Yoshifumi Takahashi

\title{
2 The Composite Pipe Construction Method
}

\subsection{What Is a Composite Pipe?}

The term "composite pipe" was first coined in the Pipe Rehabilitation Handbook published by the Japan Sewage Works Association in 2001 (JSWA, 2001). Based on its definition, the existing sewer and the renovation layer in a composite pipe are designed to resist external loads as an integrated structure, and the load-carrying capacity and durability are required to be comparable or superior to a newly-constructed sewer. In order for the existing pipe to function like a permanent member, concrete strength needs to be retained for about 50 years after completion of rehabilitation work.

To evaluate the strength of old concrete pipes in preparation for reconstruction projects, the Tokyo Metropolitan Government (TMG) dug up a total of 130 ageing pipes from 26 sewer lines over a period of two years from 1999 to 2000. A series of tests including the JIS external pressure test, uniaxial compression test and carbonation depth test were carried out on specimens taken from the dug-up pipes. Figure 2.1 shows the relationships of the cracking and fracture loads of each pipe divided by the code-specified value at the time of construction and the age of the pipe. Data points for the 60-year-old or older pipes indicate the hand-cast reinforced concrete pipes buried in the pre-war years, and those for the less-than-60-year-old pipes indicate the centrifugally-cast reinforced concrete pipes (Hume pipes: HP) buried in the post-war years. As shown, there is no clear correlation between age and strength ratio, and even if pipe deterioration occurs during a period of 70 years of service, the strength of installed pipes still satisfies the code requirements.

Figure 2.2 shows the relationship between the uniaxial compressive strength and the age of HP specimens by type of damage. As shown, the strength of buried concrete does not decrease for about 40 years. A comparison between damaged pipes and undamaged pipes reveals no significant difference in average strength, indicating that the damage was not caused by pipe deterioration.

From these test results, it can be concluded that because the initial strength of buried concrete pipes is retained even in cases of chemically induced deterioration, the concept of the composite pipe for sewer renovation is valid. 


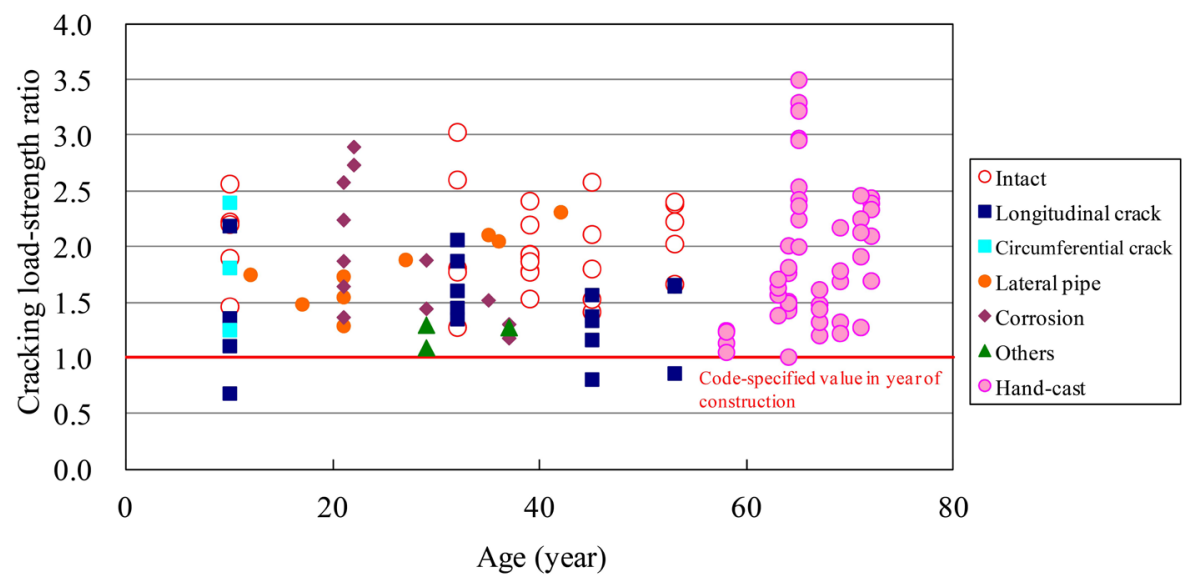

(a)

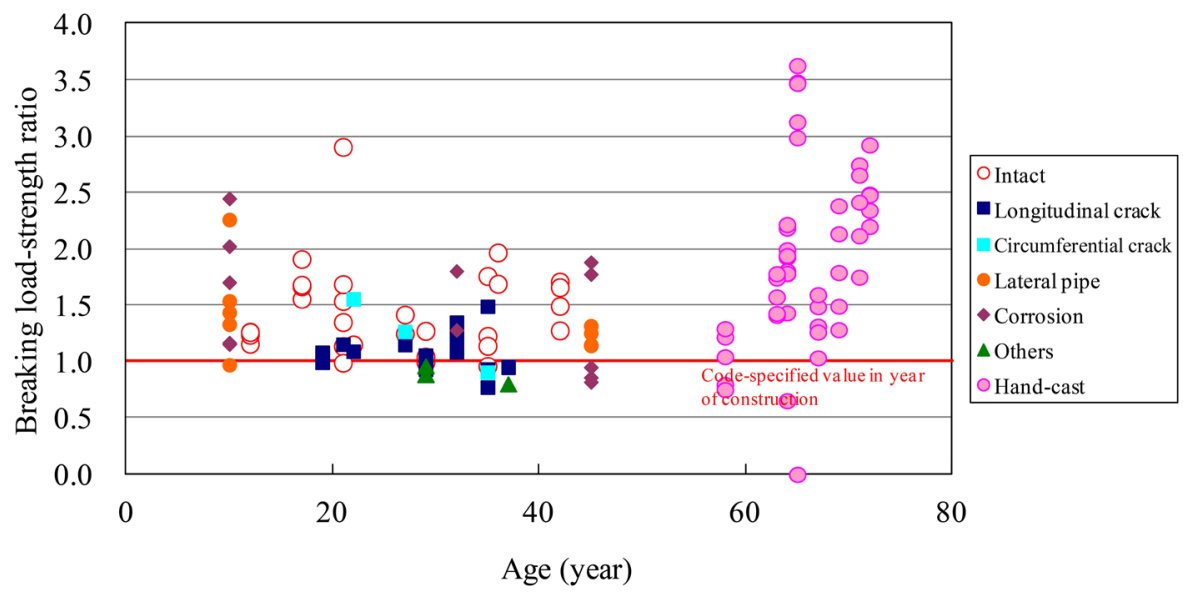

(b)

Figure 2.1: Relationship between the strength of dug-up pipes and the age: (a) cracking load; and (b) fracture load 


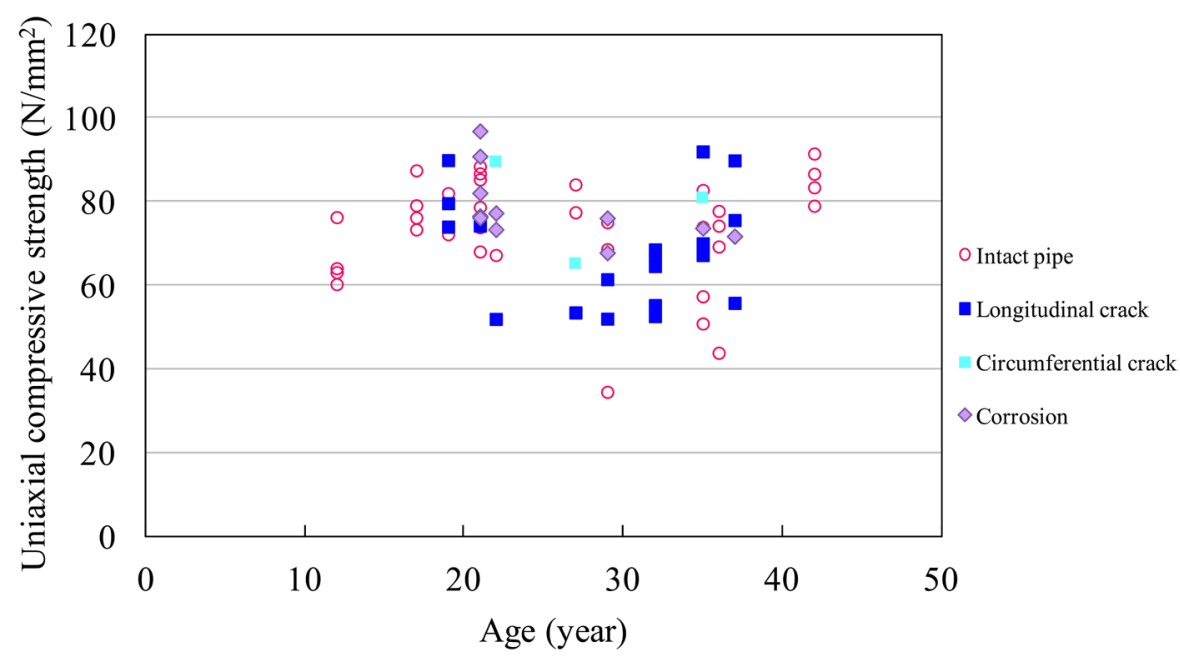

Figure 2.2: Uniaxial compressive strength of dug-up Hume pipes versus pipe age

\subsection{Classification of Pipe-Reforming Methods}

In Japan, five pipe-reforming methods for sewer renovation have been developed and employed in sewer reconstruction projects. These methods are summarised in Table 2.1. In all the methods, materials and equipment can be brought in through a manhole $60 \mathrm{~cm}$ in diameter, the existing pipe can be renovated by the trenchless method, and the gap between the liner pipe and the existing pipe is grouted with mortar so as to achieve structural integrity. In the SPR method and the Danby method, a PVC profile strip is spirally deployed by a winding machine to form a liner pipe. In the $3 \mathrm{~S}$ segment method, the PALTEM Flow-Ring method and the PFL method, plastic and high-density polyethylene pipe materials are manually assembled in the existing pipe. These five methods can be used to form not only circular pipes but also pipes with a rectangular or horseshoe-shaped cross section. Among them, the SPR method, the Danby method and the PALTEM Flow-Ring method allow the interlining to be fabricated while keeping the sewer in service. 


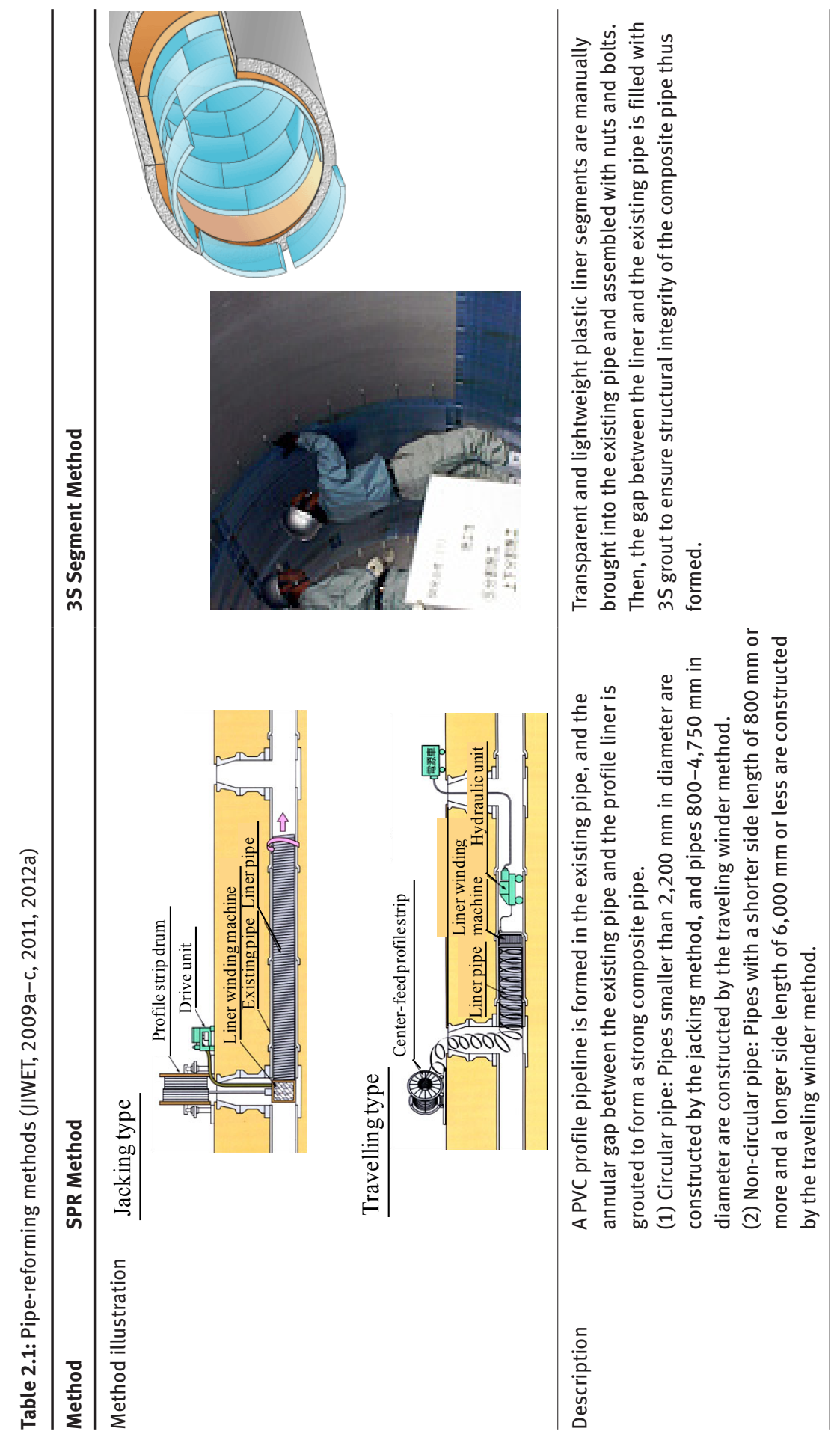




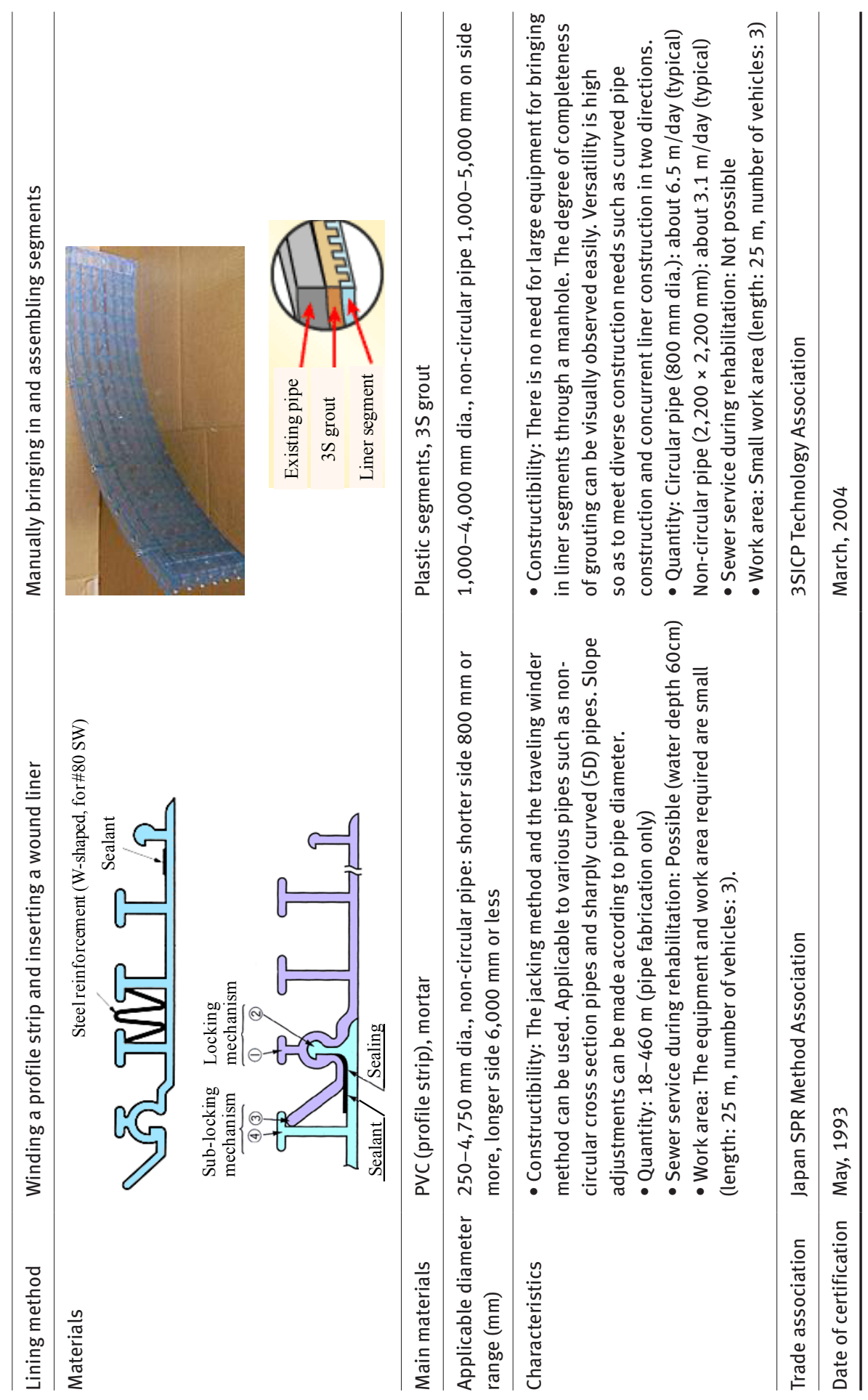




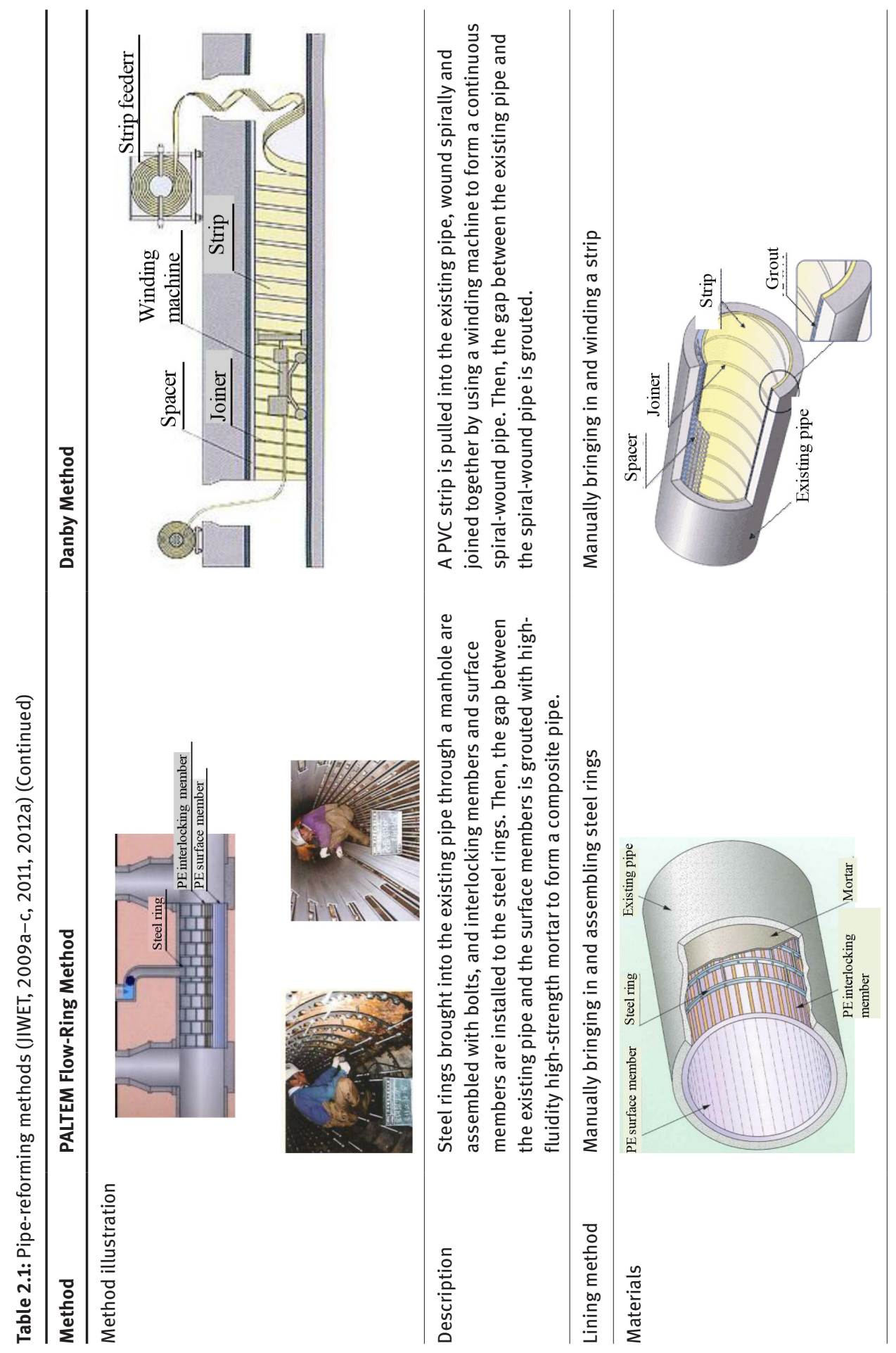




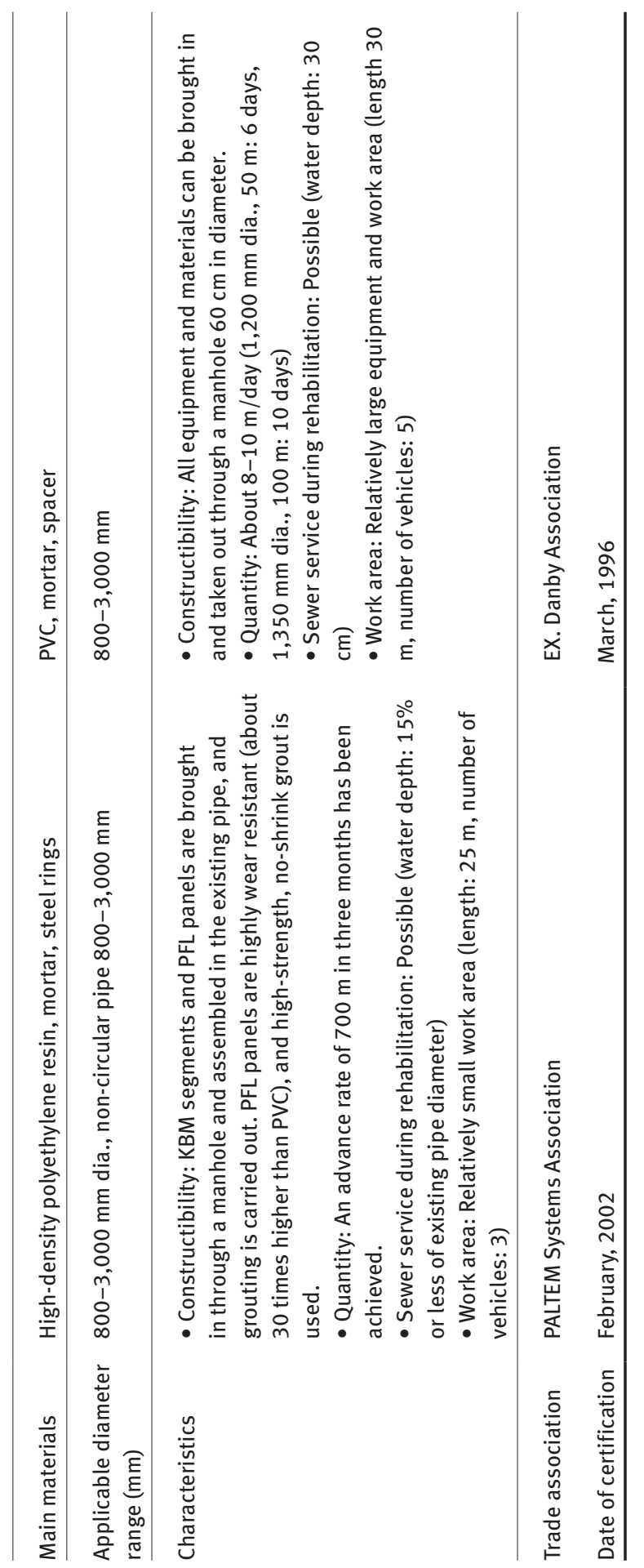


Table 2.1: Pipe-reforming methods (JIWET, 2009a-c, 2011, 2012a) (Continued)

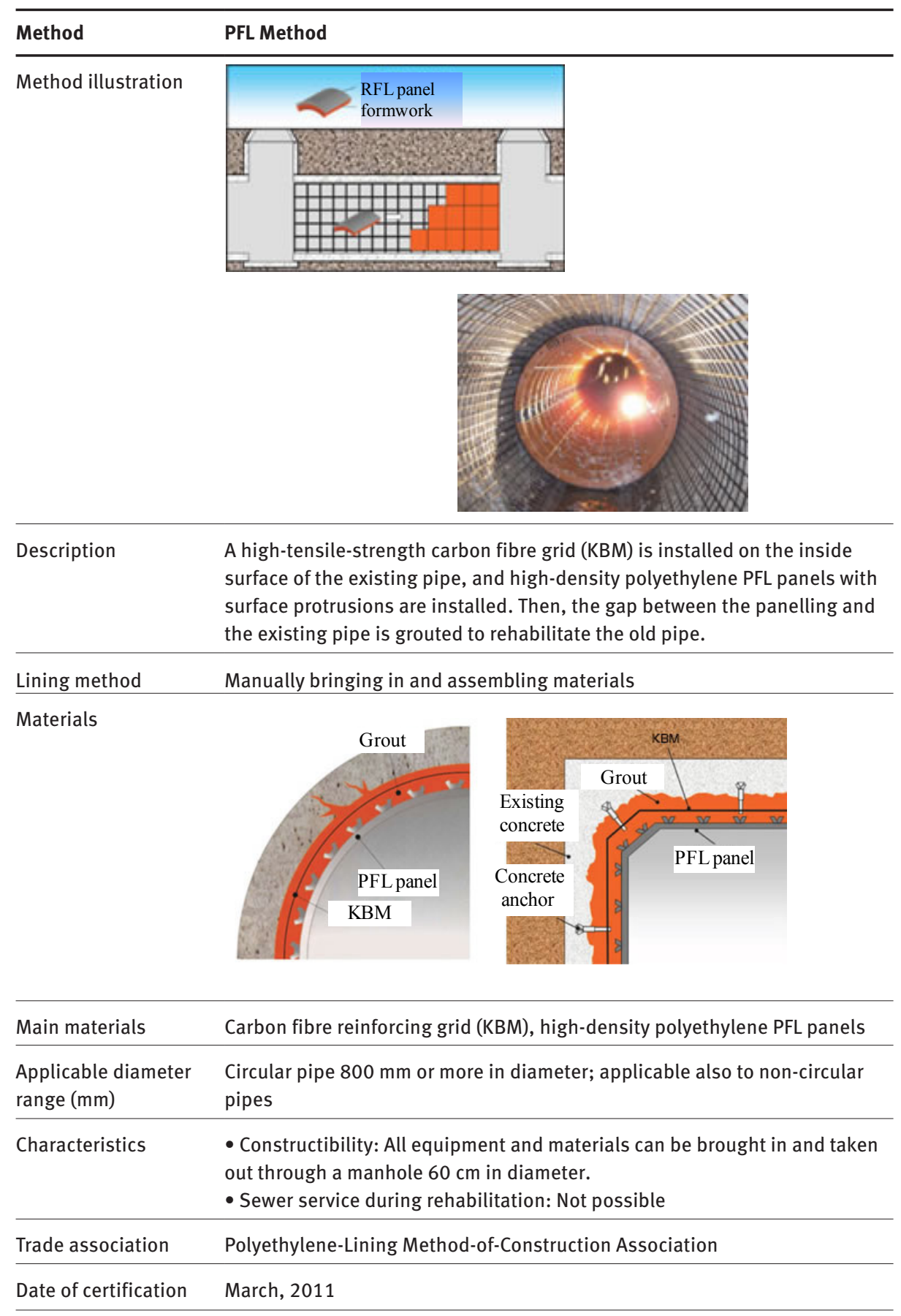




\subsection{Superiority of Composite Pipes}

Compared with independent pipes, composite pipes have the following advantages:

1. A composite pipe uses the existing pipe as part of its structure by reinforcing the existing pipe by annulus grouting. As a result of renovation, therefore, it is possible to construct a pipe structure that is stronger than an independent pipe in which the existing pipe is not expected to contribute to strength.

2. A composite pipe does not allow soil intrusion, while an independent pipe may allow soil to enter the annular gap between the old pipe and the new pipe.

3. The interlining of a composite pipe is more resistant to external chemical attack than an independent pipe because the former is covered by the existing pipe and grout.

In the case of an independent pipe placed in an old sewer pipe by the inversion method or the pull-in method, the load carrying ratio of the independent pipe increases with the progress of deterioration of the old pipe, as shown in Fig. 2.3. Eventually, the new pipe alone has to carry all loads.

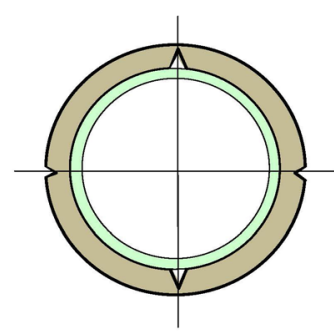

(a)

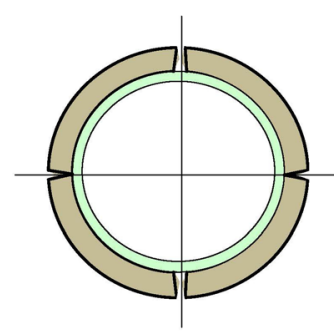

(b)

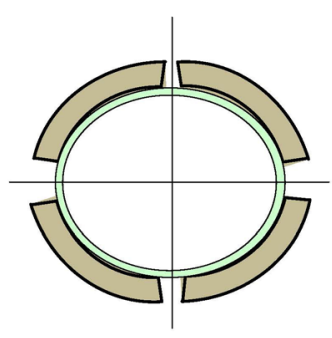

(c)

Figure 2.3: Independent pipe: (a) upon completion of rehabilitation; (b) with passage of time; (c) final stage

When designing an independent pipe, it is common practice to permit a deflection of up to $5 \%$. If, for example, the diameter of the pipe is $300 \mathrm{~mm}$, a deflection of up to $1.5 \mathrm{~cm}$ is permissible; if the diameter is $1,000 \mathrm{~mm}$, a deflection of up to $5.0 \mathrm{~cm}$ is permissible. If such deflection occurs in a sewer pipeline, the road surface may cave in or pipelines of other utilities buried over the sewer pipeline may be adversely affected. In the case of a composite pipe, in contrast, the progress of deterioration of the old pipe can be prevented because annulus grouting will likely fill the cracks in the old pipe, compensate for the decrease in pipe wall thickness due to corrosion and strengthen the pipe structure as shown in Fig. 2.4. Since the existing pipe, the 
liner pipe and the grout filling the gap between them are integrated as a composite structure (more exactly, a semi-composite structure as explained in Chapter 1), composite pipes have higher stiffness and undergo very little deflection under the applied loads. Unlike independent pipes, therefore, composite pipes are relatively free from risks associated with land subsidence.

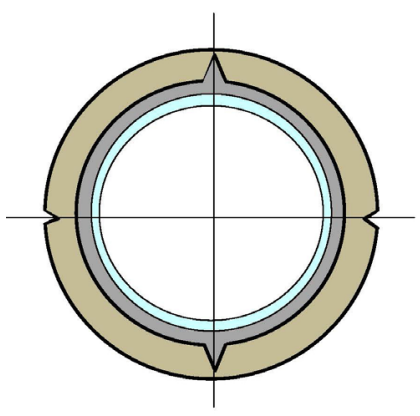

Figure 2.4: Composite pipe

If independent pipes are used for pipe rehabilitation, in many cases a gap develops between the existing pipe and the new pipe (independent pipe) because the new pipe shrinks in the existing pipe after hardening. Since no remedial or preventive measures are taken to prevent deterioration of the existing pipe surrounding the independent pipe, soil and water around the existing pipe may flow into the gap through cracks or other openings. As a result, cavities form around the existing pipe, increasing the possibility of road cave-ins. In contrast, in the case of a composite pipe, cracks in the existing pipe are filled with grout, and the gap between the existing pipe and the liner pipe is also filled with grout to ensure structural integrity. Consequently, composite pipes are basically not prone to the intrusion of groundwater and soil into the gap between the existing pipe and the liner pipe.

As shown in Fig. 2.3(c), an independent pipe installed for pipe rehabilitation comes into direct contact with the ground soil. Therefore, if there are corrosive substances in the ground, they accelerate the deterioration of the independent pipe. Composite pipes, however, are free from such risk because the liner pipe is doubly protected by the existing pipe and the grout.

\subsection{The SPR Method}

To illustrate technical developments in sewer renovation using the composite pipe methodology in Japan, this section briefly describes the SPR method developed by a joint team of three companies including the authors over a period of three decades. 
The development of the SPR method dates back to 1984, when Adachi Construction and Industry Co., Ltd., one of the developers of the SPR method, proposed a technical plan for rehabilitating an old pipeline by spirally winding a steel strip. At around the same time, Sekisui Chemical Co., Ltd., another developer, entered into a licensing agreement with Rib Loc, an Australian company, for its PVC profile strip products, and improved their quality and shapes to enable them to be used as liner pipes for the rehabilitation of old sewer pipes. The first field test was carried out in Yokohama City in a sewer rehabilitation project. In 1985, the Tokyo Metropolitan Sewerage Service Corporation (TGS) joined the development team, since then the three companies have been jointly developing the technologies concerned.

Initially, a method called the jacking method was used for lining construction. As shown in Fig. 2.5, in the jacking method, a jack-type winder is installed in a manhole, and a profile strip fed from a profile strip drum placed on the ground surface is pushed into the existing pipe while the winder performs spiral winding and interlocking operations (refer to Photos 2.1 and 2.2). At first, this method was applied to smalldiameter pipe rehabilitations. In 1987, it was used for renovating pipes of 250 to 800 $\mathrm{mm}$ in diameter, and in 1988 for pipes of 900 to 1,200 $\mathrm{mm}$ in diameter.

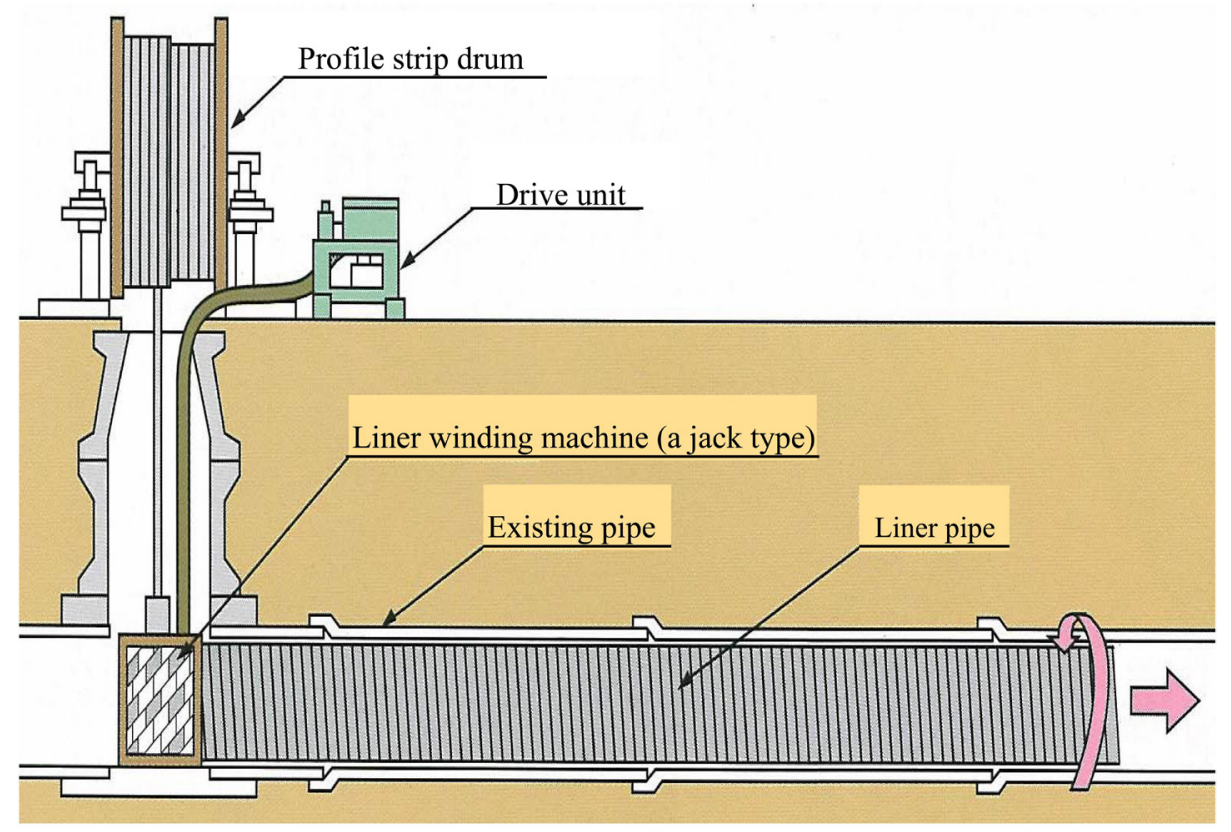

Figure 2.5: Construction of liner pipe by spiral winding using a jacking method 


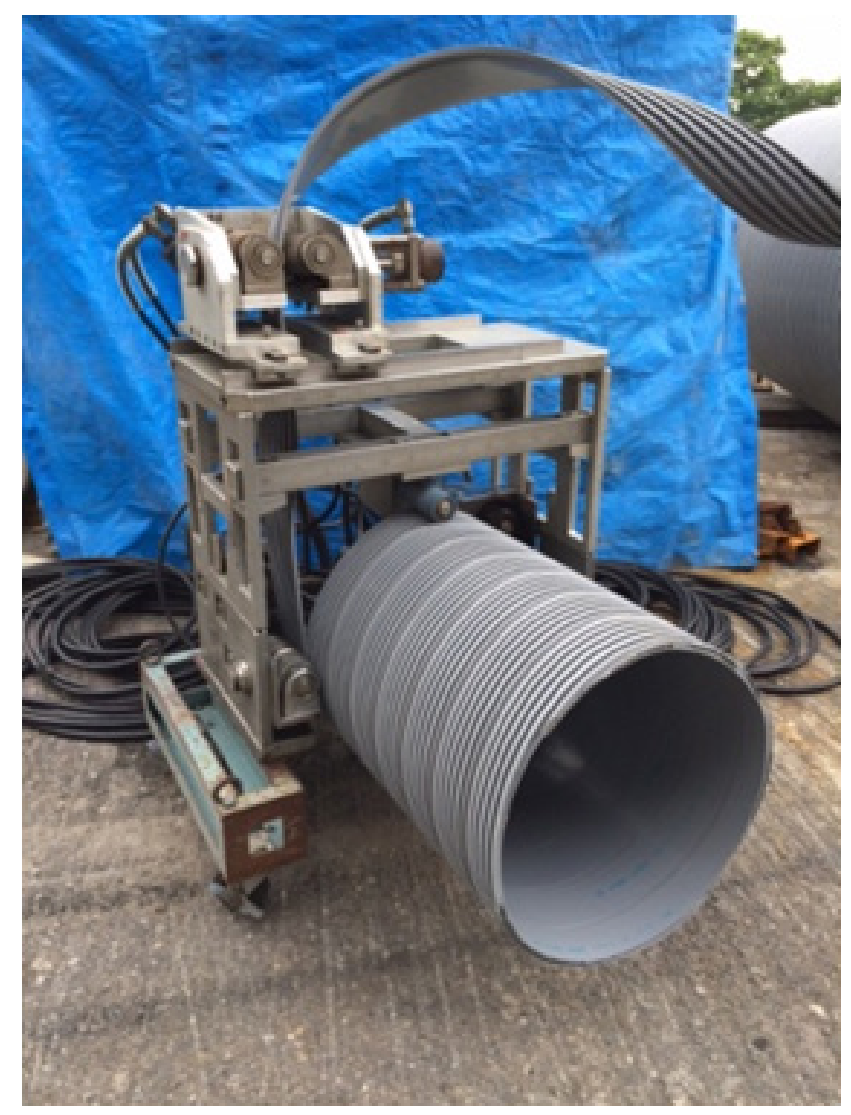

Photo 2.1: A jack type winding machine
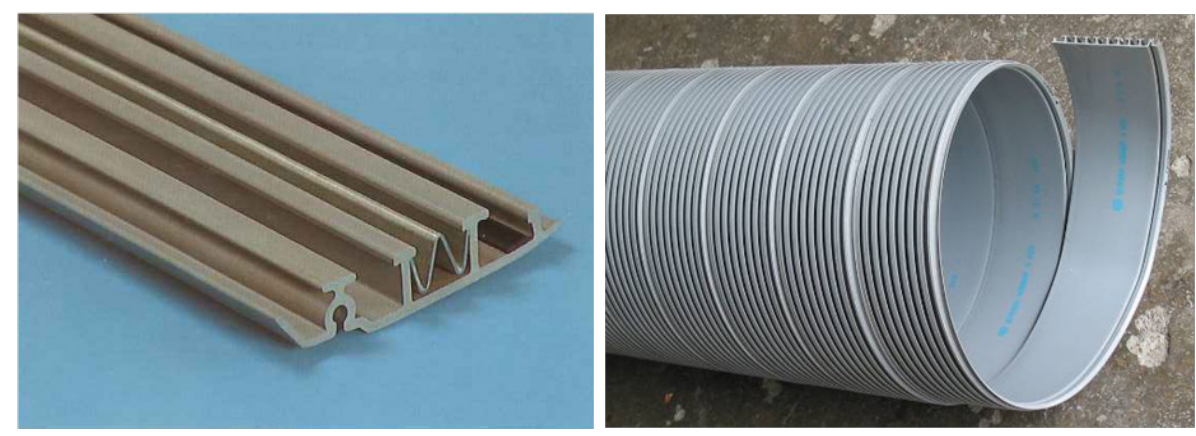

Photo 2.2: PVC profile strips 
Later, as experience was accumulated in rehabilitating medium- and small-diameter pipes, demand grew for large-diameter-pipe rehabilitation technology. In order to meet this demand, the Super SPR method was developed for rehabilitating largediameter pipes ranging in diameter from $1,650 \mathrm{~mm}$ to $2,200 \mathrm{~mm}$. The Super SPR method was made possible by a new technology for installing steel reinforcements to conventional profile strips. The Super SPR method was approved for use in statesubsidized projects for large-diameter sewer restoration following the Great Hanshin Earthquake and was used in many projects in the subsequent years.

In the jacking method of liner making, a spirally wound liner pipe is rotated and pushed into an existing pipe. Naturally, there is a limit to the pipe length and diameter to which the method can be applied, due to the limitations in both the driving power and stiffness of the liner. Therefore, the development team began to develop a linermaking method using a travelling winder capable of advancing in the existing pipe while winding a profile strip, as shown in Fig. 2.6 and Photo 2.3. In 1996, the newly developed method was used to renovate an ageing sewer of 1,200 $\mathrm{mm}$ in diameter.

Old sewer mains include various non-circular sewers such as rectangular, horseshoetype and covered sewers, and the team began to develop a method for renovating such sewers. In 1998, a method called the Free Cross Section SPR method began to be used in the Tokyo area, and has since been used to rehabilitate various sewers including a rectangular sewer $6 \mathrm{~m}$ wide and $3 \mathrm{~m}$ high. As shown in Photo 2.4, it is now possible to rehabilitate pipes of any shape. Major factors that have made this possible include the availability of prefabricated frames designed to fit into existing pipes and the shaperetaining effect of the reinforcing steel members installed in the profile strips.

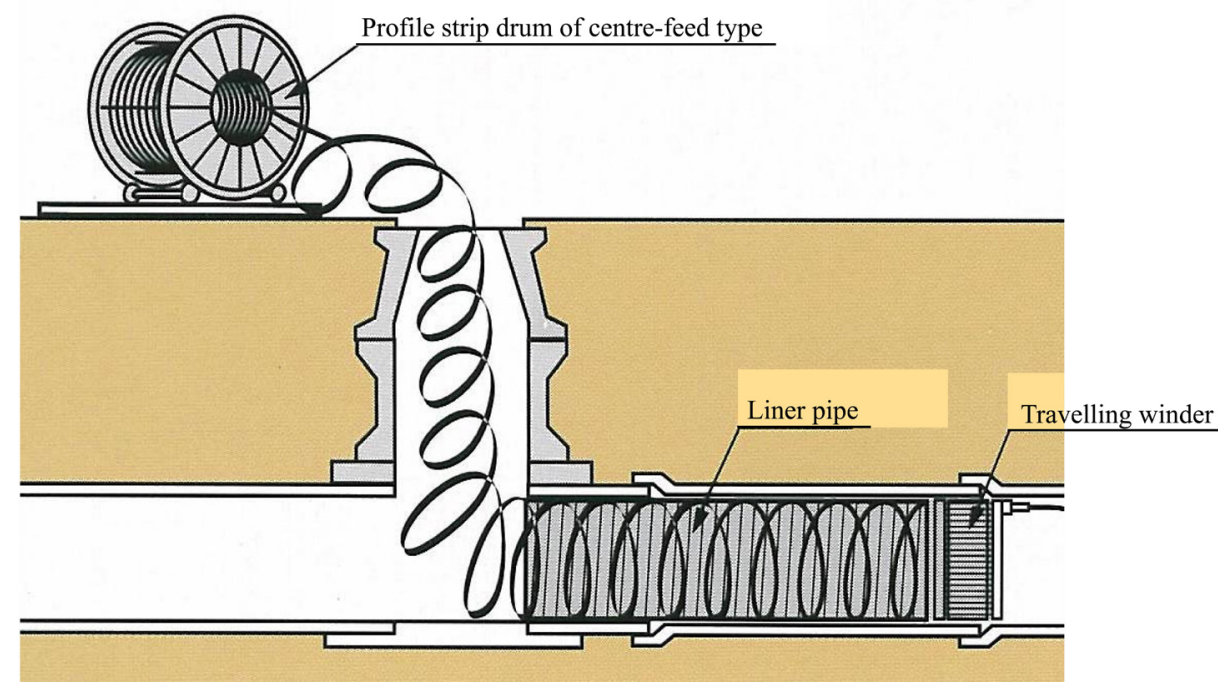

Figure 2.6: The travelling winder method of liner making 


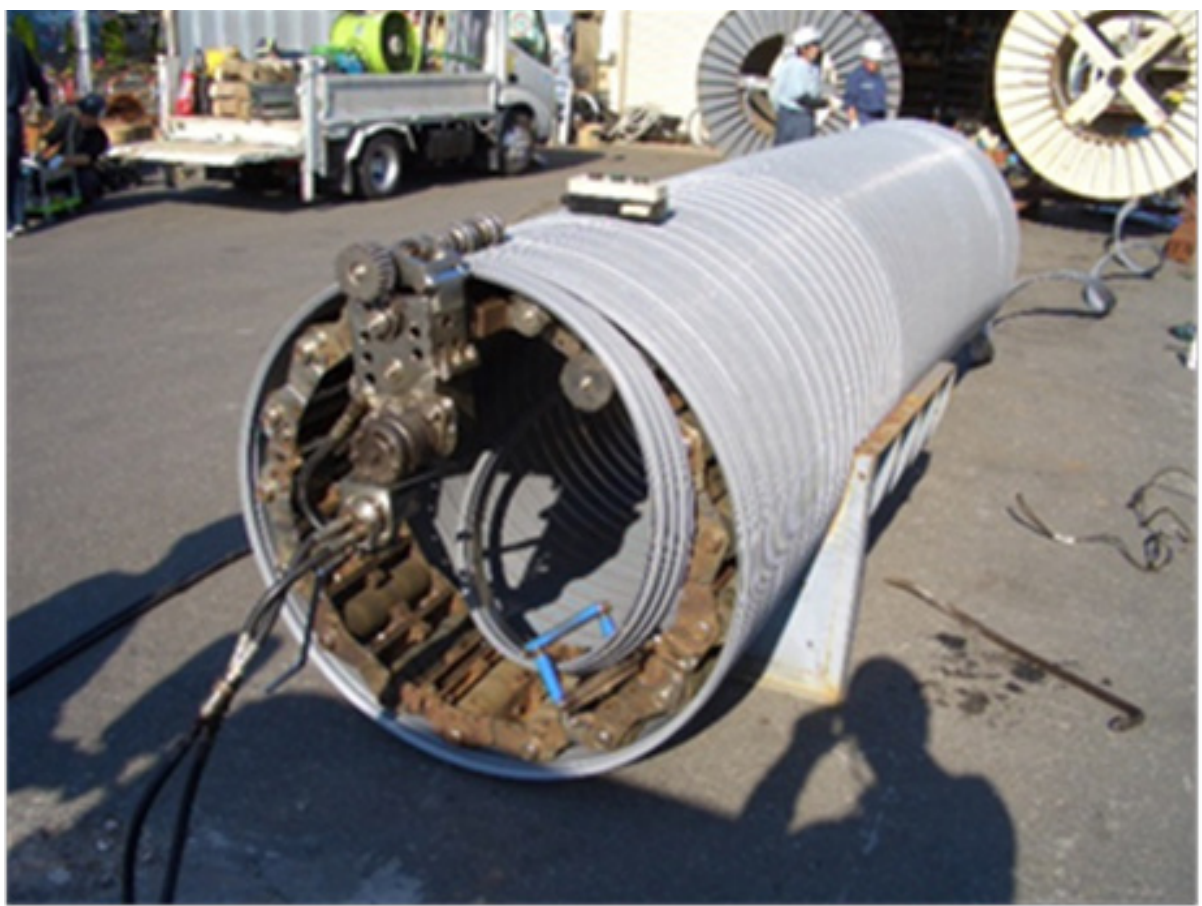

Photo 2.3: A travelling type winding machine
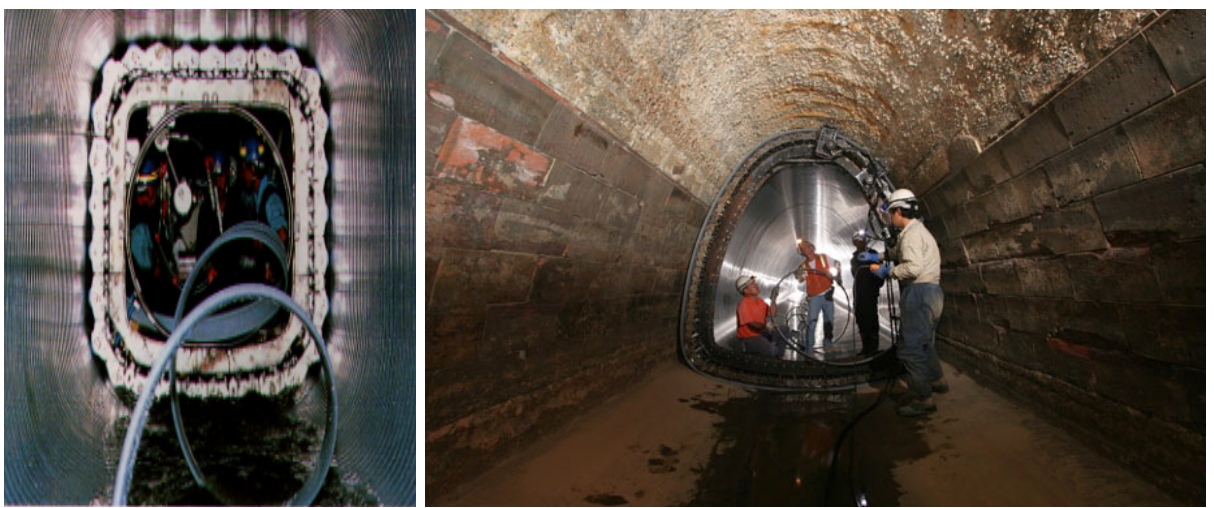

Photo 2.4: Free cross section SPR method

Concurrently with the development of the winders and liner pipe materials mentioned above, work continued on developing a special mortar to be injected into the gap between the existing sewer and the liner pipe. The requirements for such grouting mortar included not only high strength but also fluidity, segregation resistance, 
shrinkage resistance and the bond strength with existing concrete. Improvements were made through many mix design tests, laboratory element tests, laboratory fullscale model tests and in situ tests, and currently four types of grout materials are used to meet project-specific needs. The strongest mortar, No. 4, has a compressive strength of $55 \mathrm{~N} / \mathrm{mm}^{2}$.

As shown in Table 2.1, with the SPR method, it is possible to keep the host pipe in service, allowing a water depth of up to $60 \mathrm{~cm}$. The construction cost of the SPR method is about half that of cut-and-cover construction. Because of these advantages, the SPR method is the most widely used pipe-reforming method of sewer renovation in Japan. Figure 2.7 shows the length of sewers rehabilitated by the SPR method by fiscal year: the total length rehabilitated by the method by 2011 was $762 \mathrm{~km}$.

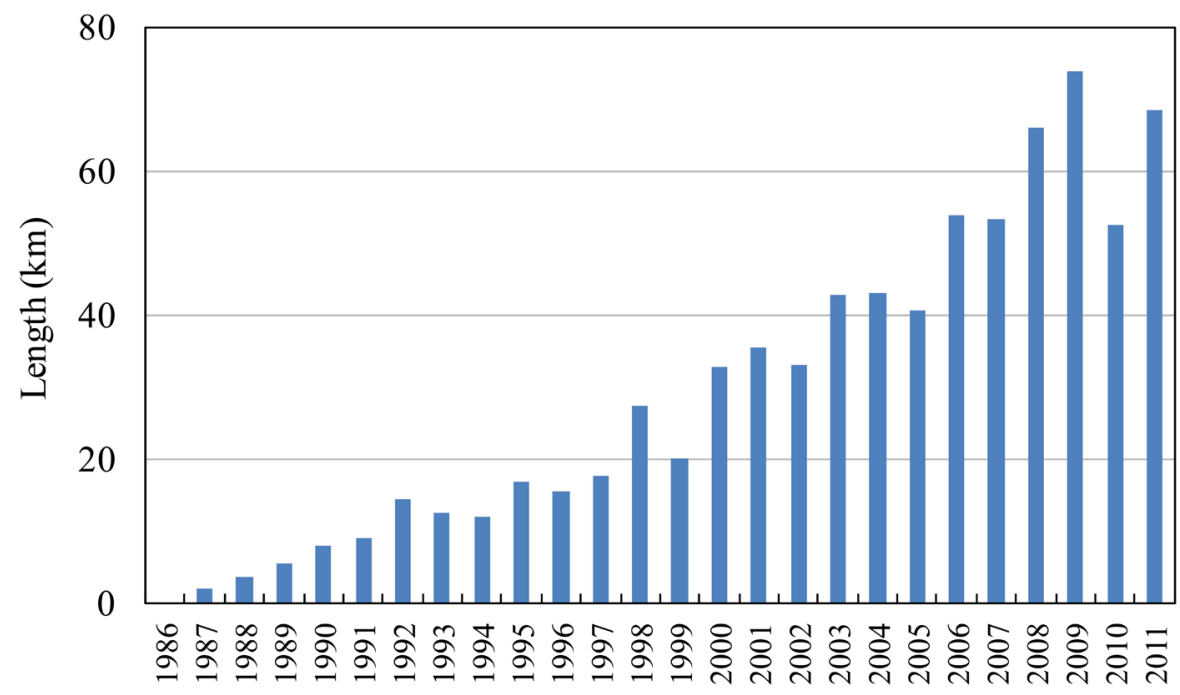

Fiscal year

Figure 2.7: The length of ageing sewers renovated by the SPR method each fiscal year since 1986

At present, the development team is studying ways to solve challenges such as dealing with sharp curves and improving economy and safety. For sharp curves, profile strips capable of expansion and contraction in the width direction have been developed. As a result, it is now possible to deal with a radius of curvature of $R=5 D$ ( $D$ : existing pipe diameter), as shown in Fig. 2.8 and Photo 2.5. 


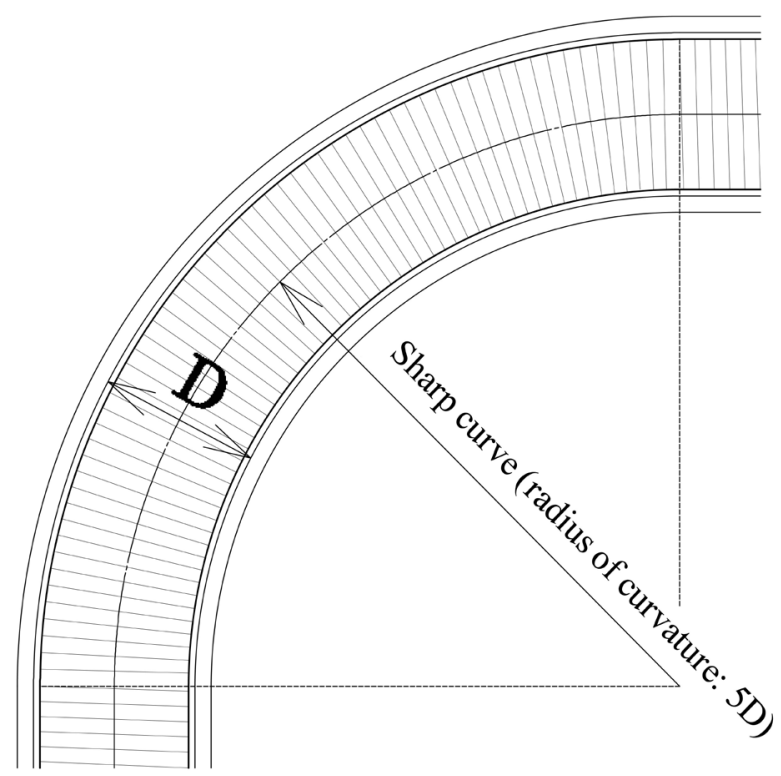

Figure 2.8: Dealing with curve construction

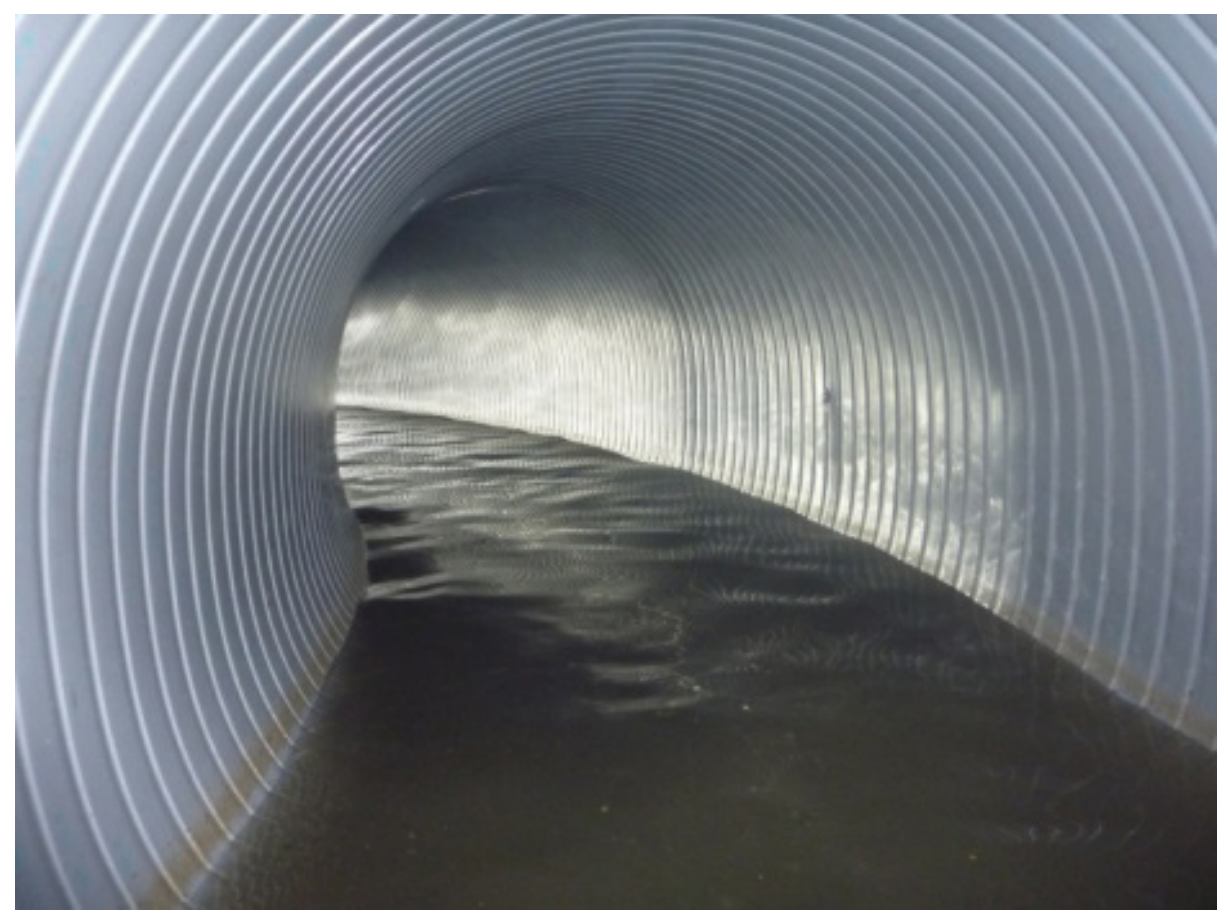

Photo 2.5: Curve construction by the SPR Method 


\subsection{Renovation Construction of Composite Pipes: From Investiga- tion to Construction}

This section briefly explains the stages of investigation, design and construction in a sewer rehabilitation project with examples of the SPR method.

\subsubsection{Investigations necessary for composite pipe design}

\subsubsection{Medium- to large-diameter pipe}

In a project involving a medium- to large-diameter pipeline of man-entry size (inside diameter: $800 \mathrm{~mm}$ or more), four types of investigations are conducted:

1. Drainage area investigation

2. Surveying

3. In-pipe investigation

4. Environmental investigation

The drainage area investigation involves collecting reconstruction-related information, specifically information on the conditions of and future plans for the drainage area and sewer mains concerned. Field reconnaissance is also conducted to investigate the conditions around the sewer. Surveying involves collecting information on the sewer specifications, ground elevation, earth cover, etc. in the area over the sewer line. The in-pipe investigation includes visual observation, internal cross-sectional measurement, wall flatness investigation and existing pipe structure investigation. The environmental investigation is conducted to collect information on the flow rate, flow velocity and other site conditions that may affect pipe winding operation such as step displacement, heaving and differential settlement and decide on actions to take during construction.

The structural investigation conducted on the existing pipe as part of the in-pipe investigation necessary for structural analysis and renovation design is briefly explained below, with the two ageing sewers shown in Photo 2.6 as examples under investigation.

\section{1) Concrete strength investigation}

Core samples are taken from the existing sewer, and a compressive strength test is conducted. Prior to core sampling, the locations of reinforcing bars and the degree of damage are investigated through visual inspection and reinforcing steel detection, and core sampling locations are determined, taking into consideration the minimisation of adverse effects on safety and the number of samples needed to achieve the required level of accuracy. Specimens for compressive strength testing are taken from noncarbonated regions.

The number of core samples taken should be limited for the safety of the existing pipe. As a complementary test, therefore, a rebound test using a Schmidt hammer is also conducted. Photos 2.7 to 2.10 show how the core sampling, Schmidt hammer test and compressive strength test are conducted. 


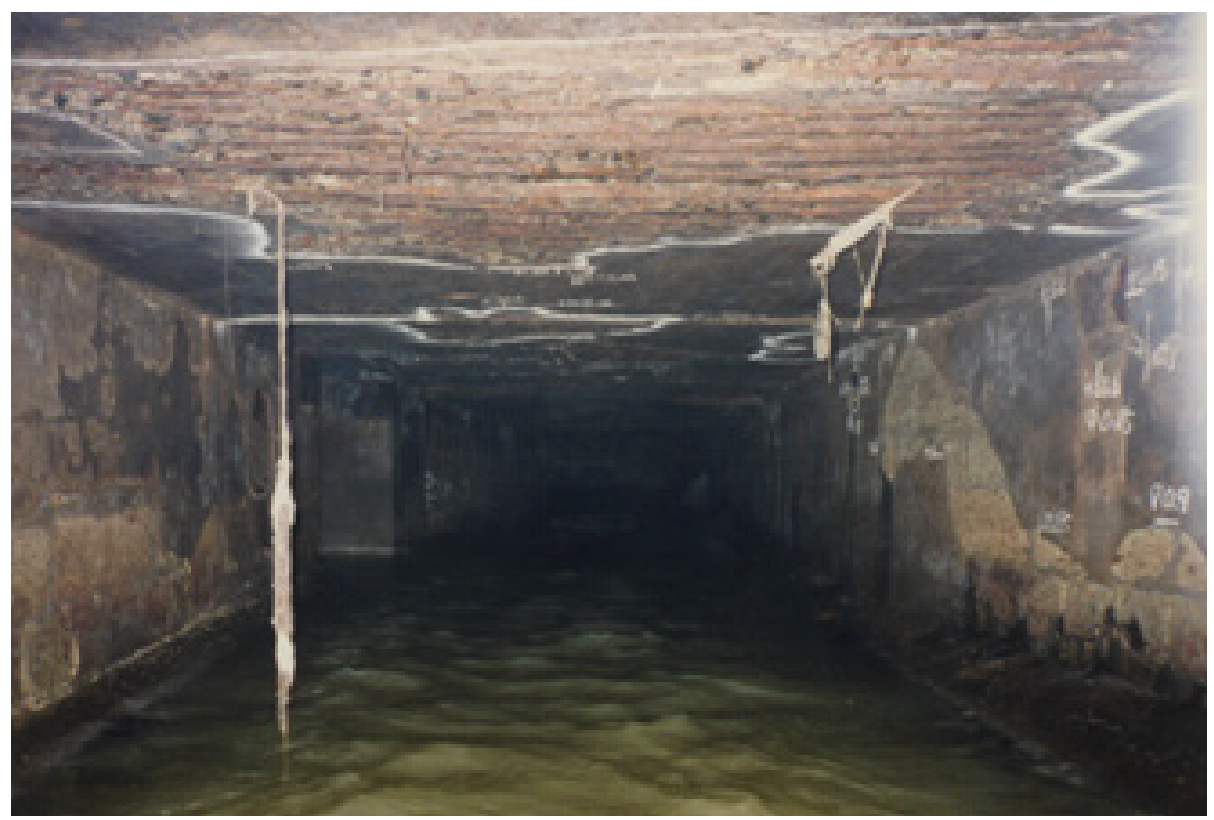

(a)

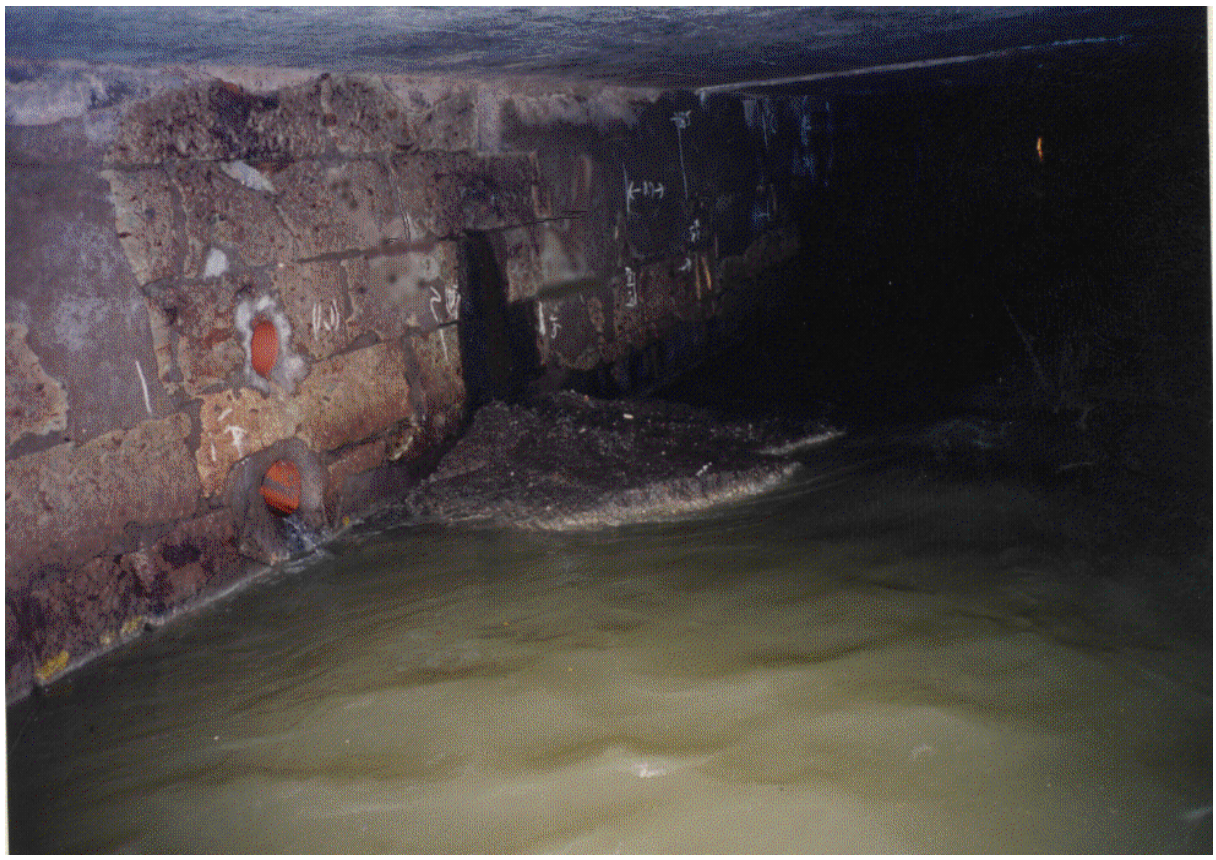

(b)

Photo 2.6: Ageing sewers: (a) rectangular sewer with exposed reinforcing bars; and (b) covered stone masonry sewer with accumulated sediment 


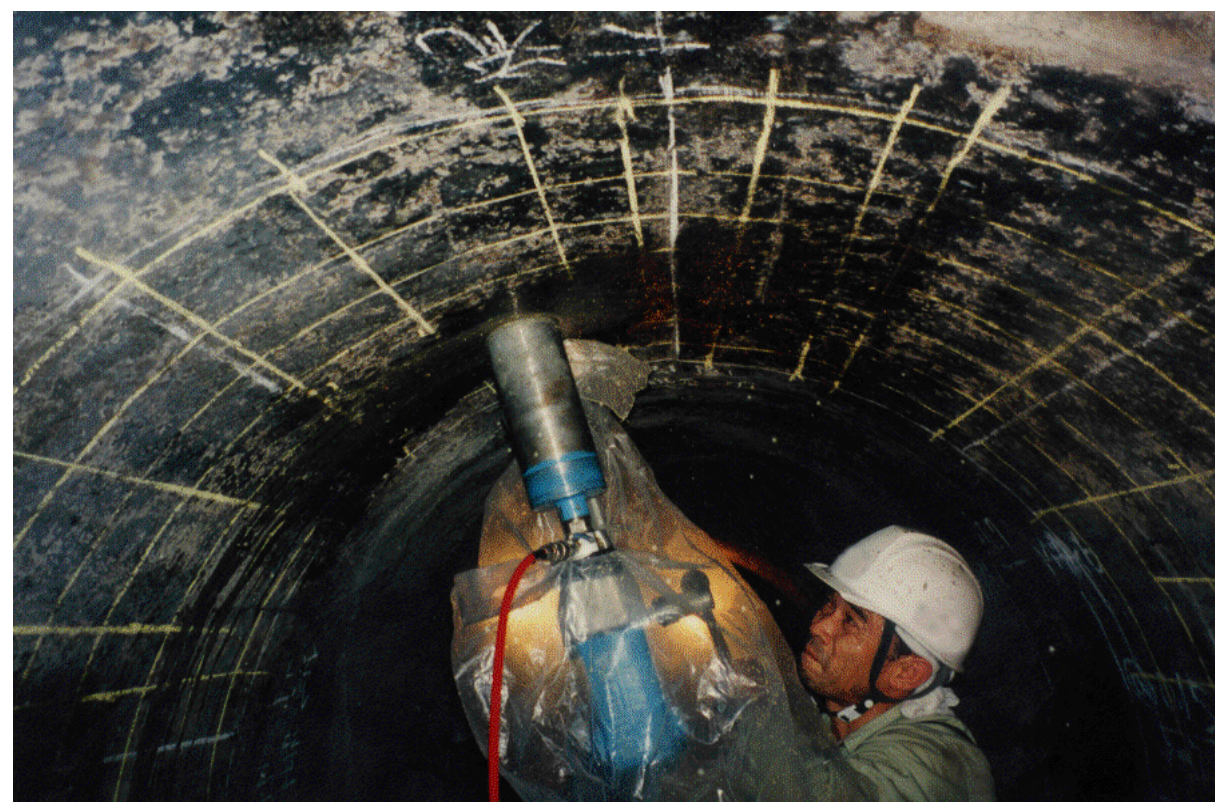

Photo 2.7: Core boring

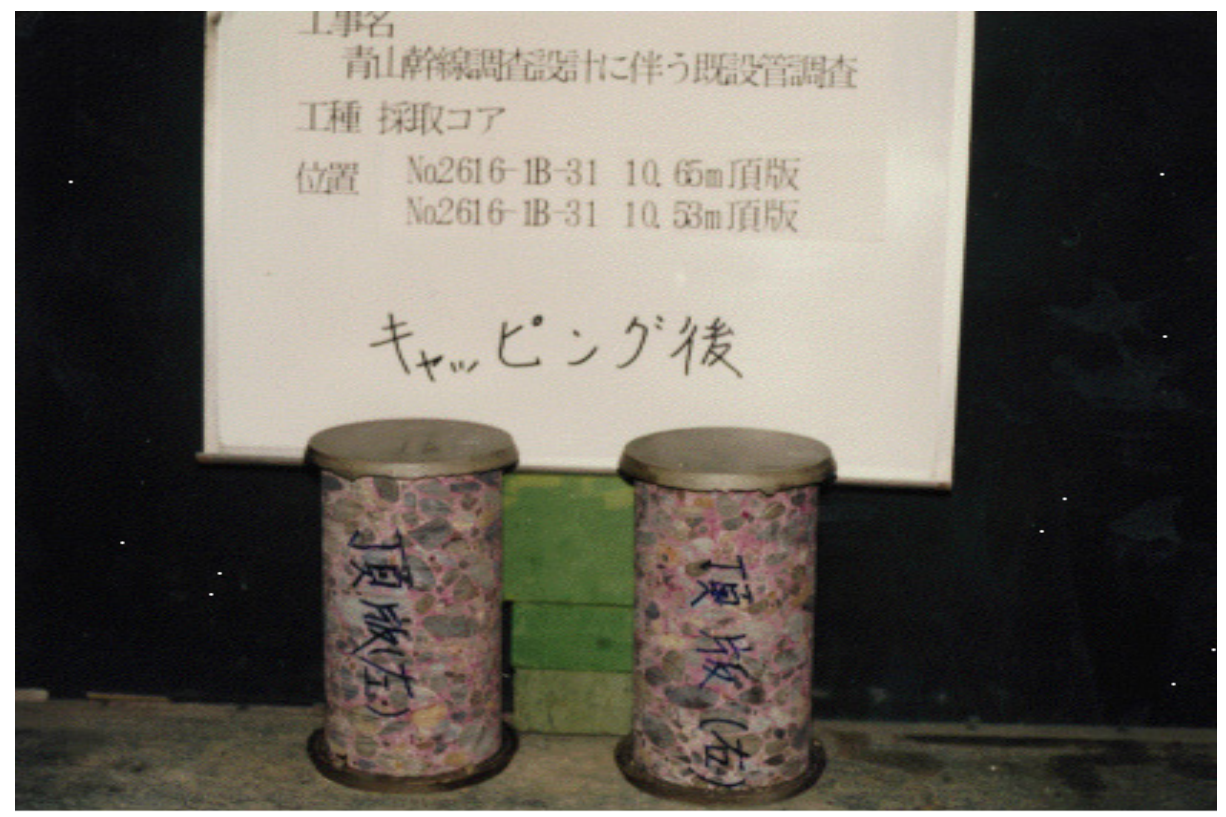

Photo 2.8: Core samples 


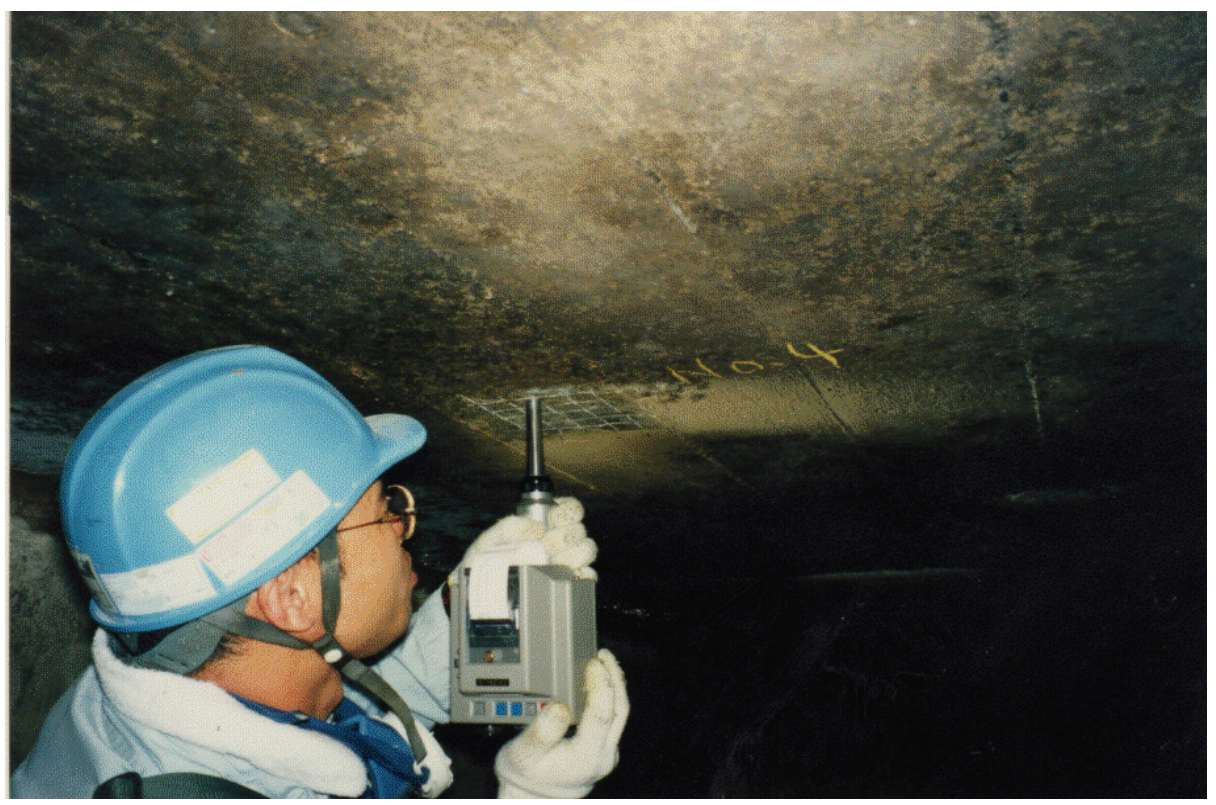

Photo 2.9: Rebound test

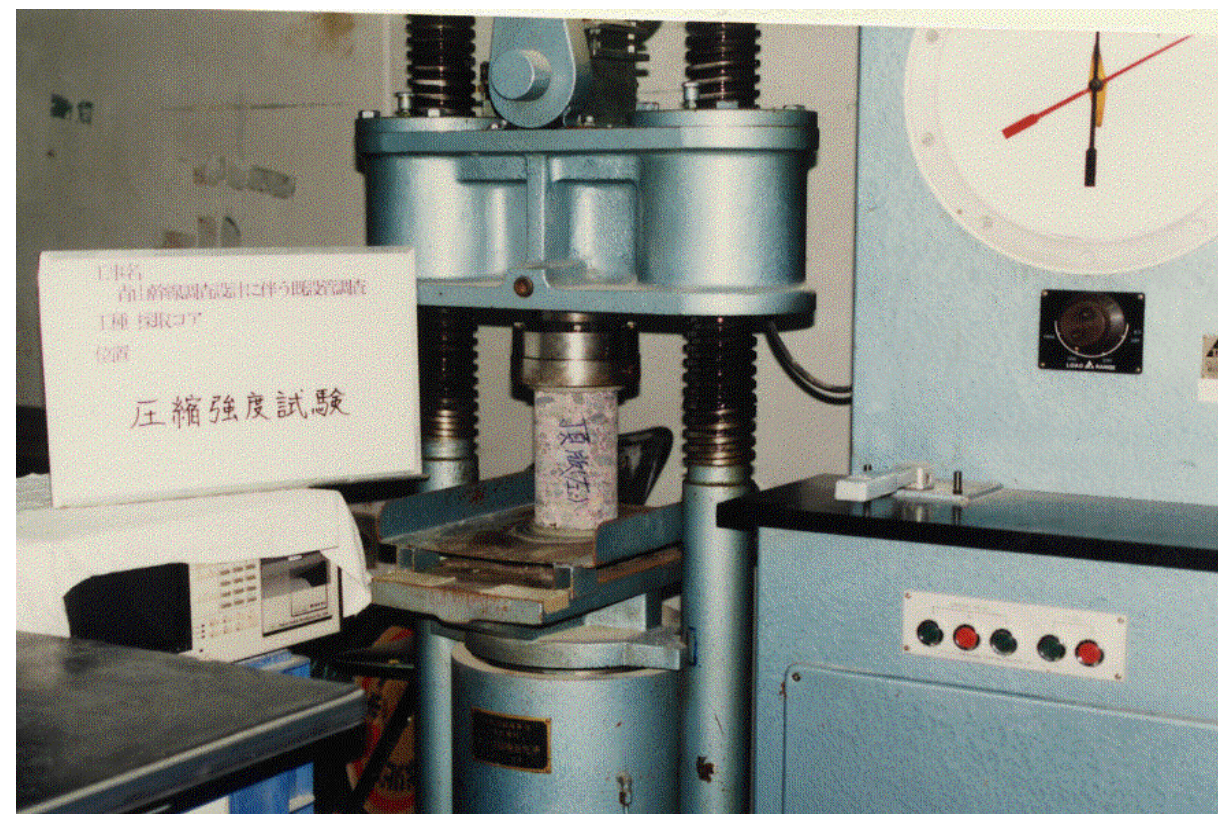

Photo 2.10: Compressive strength test 
2) Carbonation test

A carbonation test is conducted for the purpose of investigating the carbonation depth of the existing concrete. The test can be conducted by the scraping method or the coring method. In the scraping method, concrete in an area measuring about $200 \mathrm{~mm}$ by $200 \mathrm{~mm}$ is scraped off from the surface until reinforcing bars are exposed, and a $1 \%$ phenolphthalein solution is sprayed to the scraped area. Since the depth at which a red-purple colour does not appear indicates the carbonation depth, the distance from the concrete surface to that point is measured. Photos 2.11 and 2.12 show how the scraping and measurement are carried out.

In the coring method, each core is immediately washed with water after it is extracted. After the core is drained, a $1 \%$ phenolphthalein solution is sprayed. The distance from the concrete surface to the depth at which the colour of the concrete turned red-purple is measured with a ruler. Photos 2.13 and 2.14 show how this investigation is carried out.

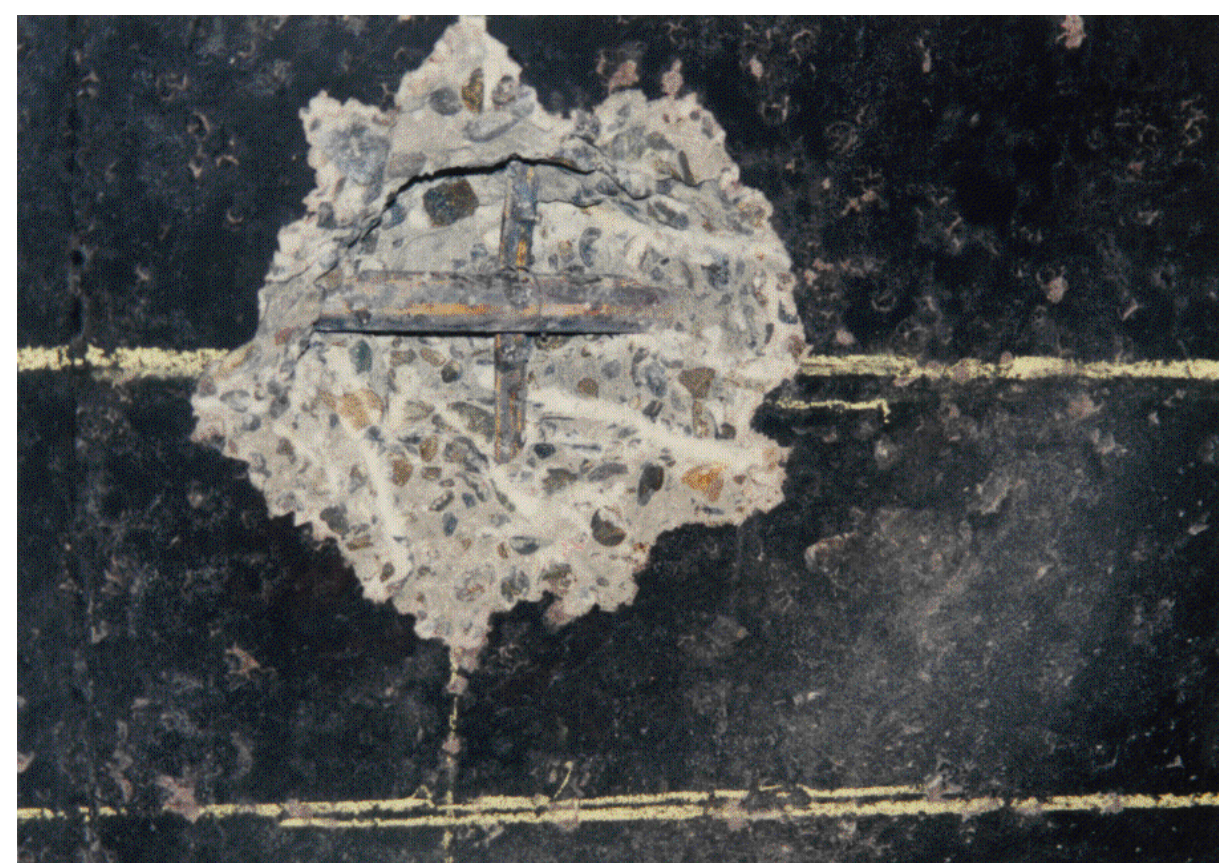

Photo 2.11: Scraping

3) Rebar arrangement investigation

The reinforcement arrangement is investigated by using a combination of two methods: the electromagnetic wave method and the electromagnetic induction method. 


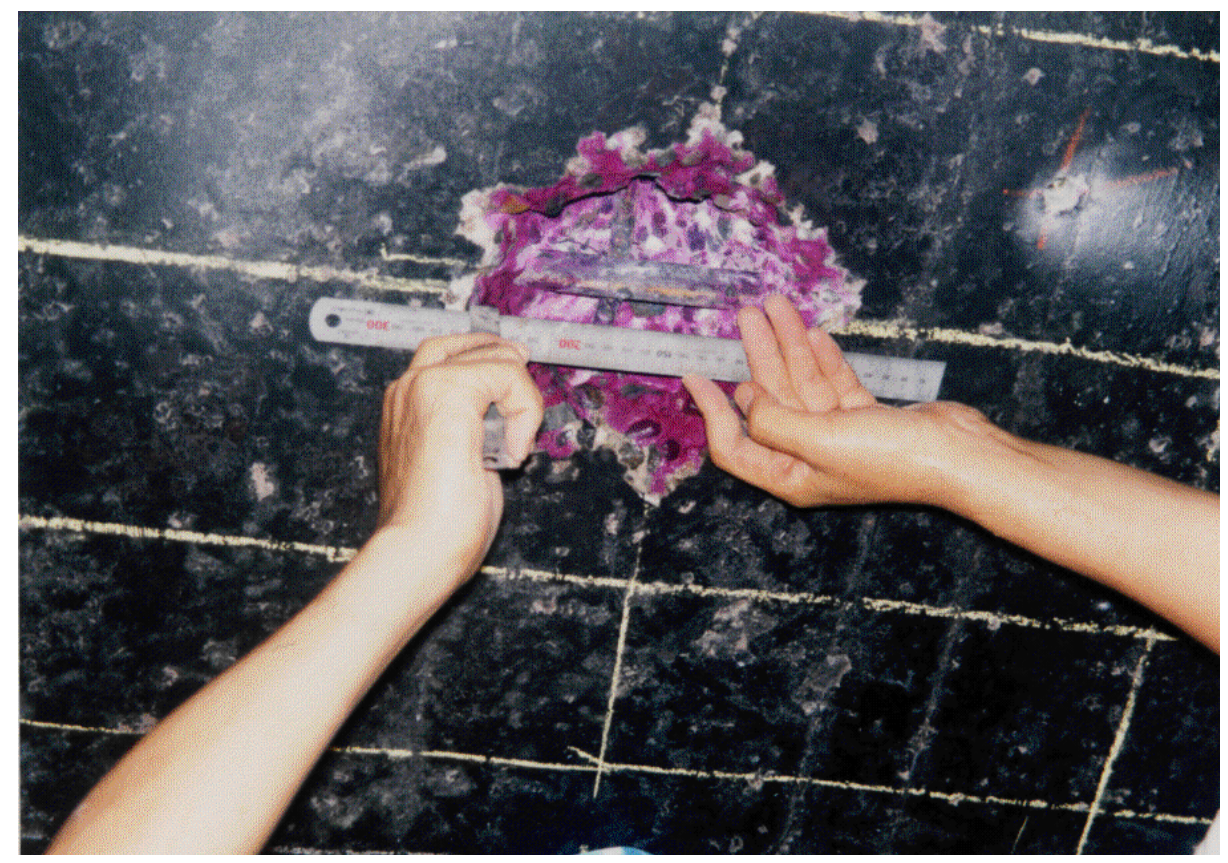

Photo 2.12: Measuring the carbonation depth

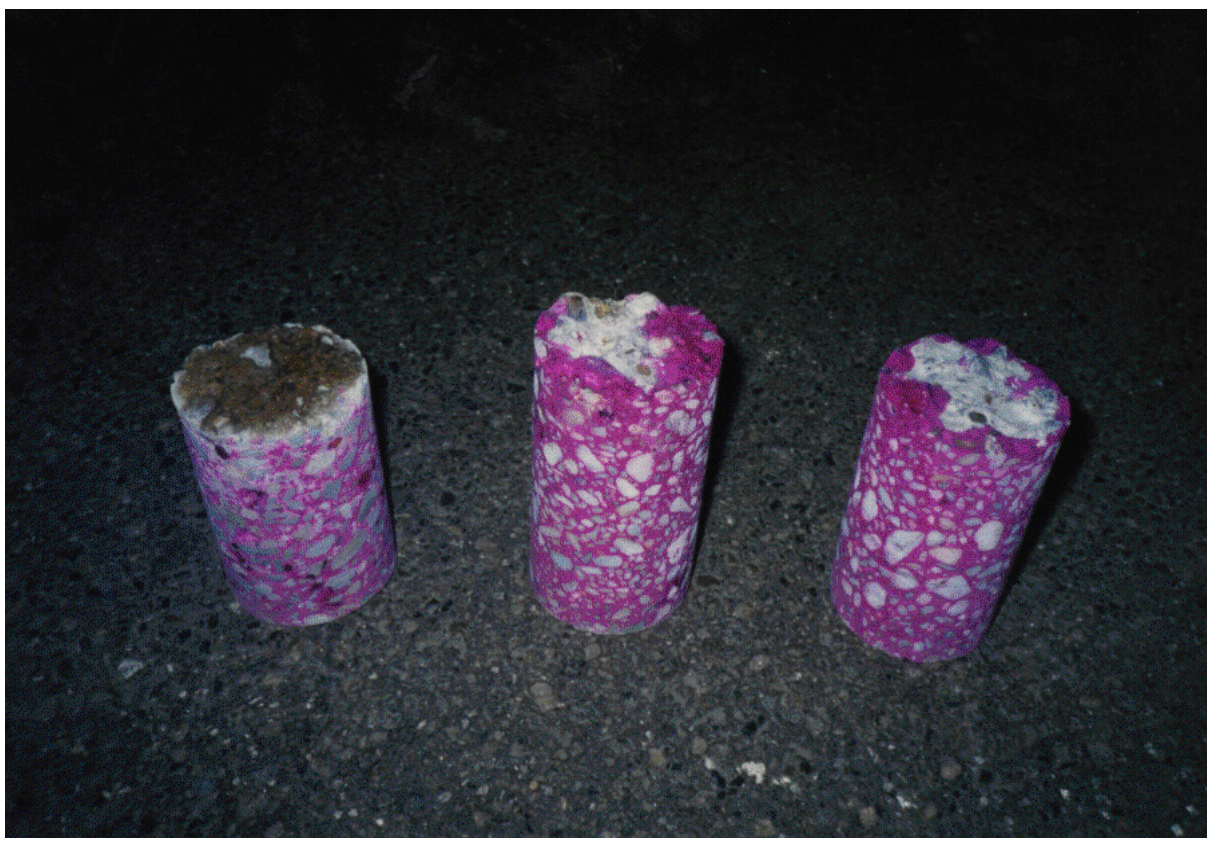

Photo 2.13: Cores for carbonation test 


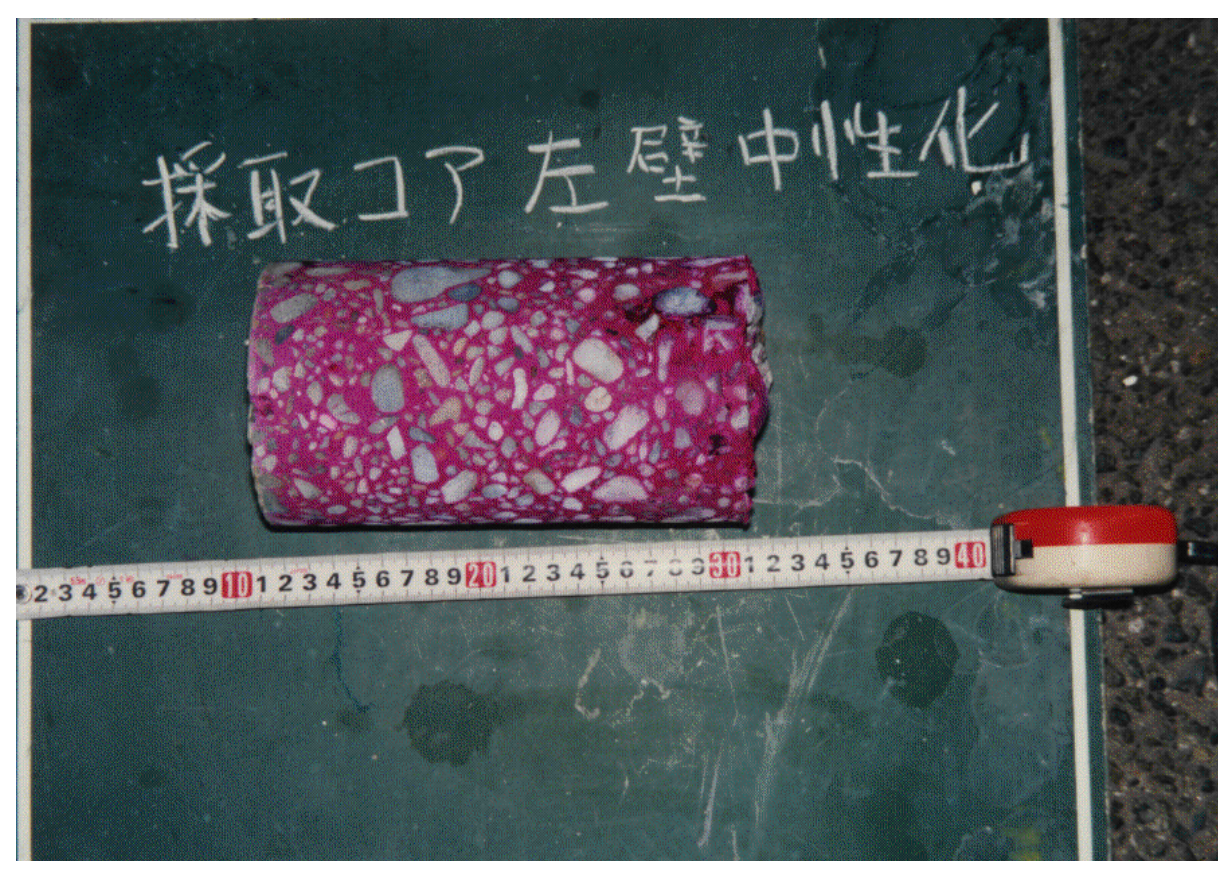

Photo 2.14: Measurement of carbonation depth

(a) Reinforcing bar detection by the electromagnetic wave method

From the concrete surface, the reinforcing patterns in the upper slab and the side walls are investigated, and reinforcement drawings are prepared. As a measuring instrument, a reinforcing bar detector, $\mathrm{RC}$ radar, is used.

To achieve high resolution at shallow depths (about $30 \mathrm{~cm}$ or less) in the concrete, the reinforcing bar detector uses pulse waves with a pulse width of about one nanosecond (1/1,000,000,000 second) for transmission. Equipped with an integrated transmit/receive antenna and capable of moving along the concrete surface, the detector can also be used to measure the spacing of reinforcing bar. Photo 2.15 shows reinforcing bar detection by the electromagnetic wave method.

(b) Reinforcing bar detection by the electromagnetic induction method

To check whether there are reinforcing bars on the outer side of an ageing pipe, reinforcing bar detection is carried out by the electromagnetic induction method at the holes from which cores were extracted. This method utilises the large difference in magnetic permeability between concrete and steel. Compared with the relative magnetic permeability of concrete which is approximately 1 , reinforcing bars are ferromagnetic and have a relative permeability as high as several dozen to several hundred. 

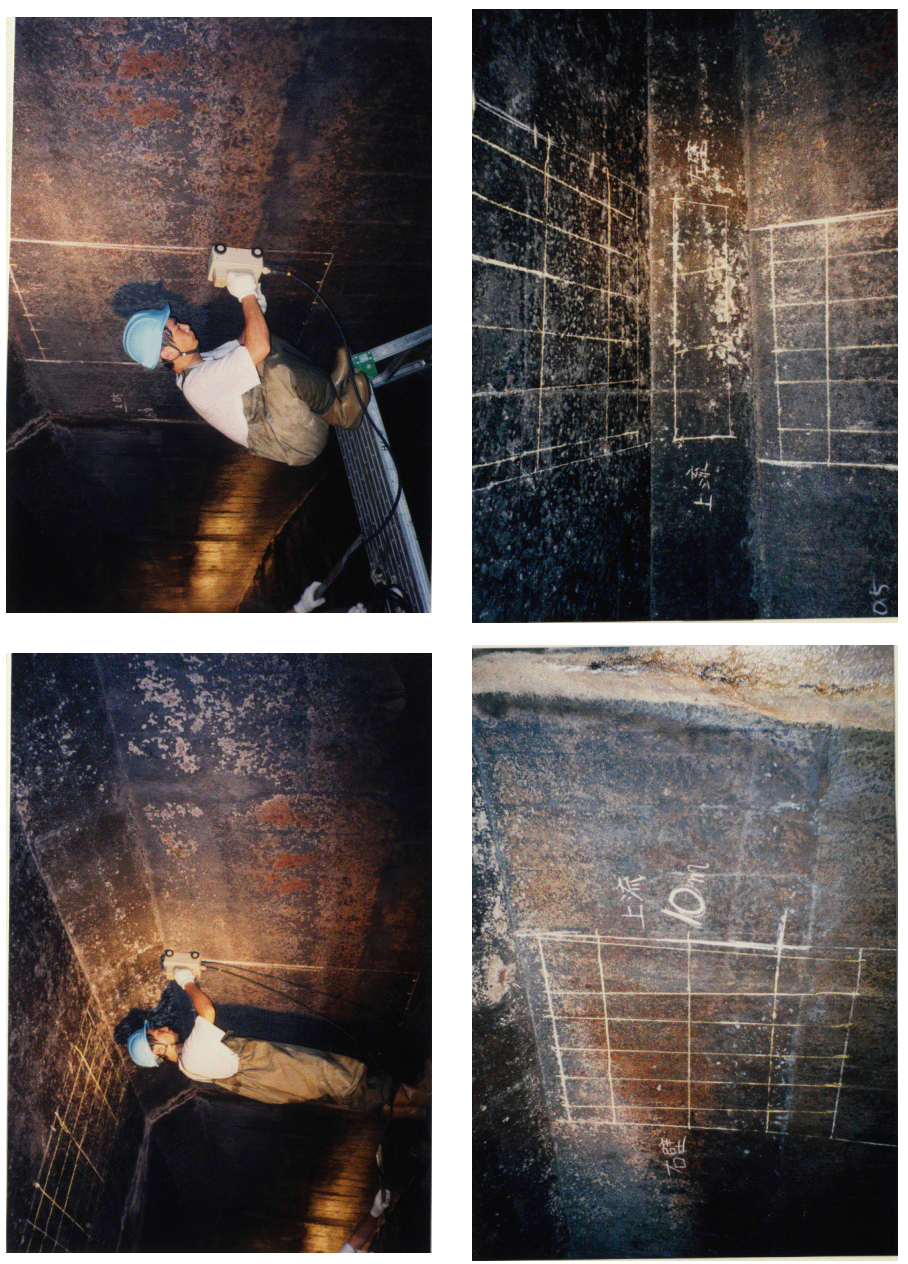

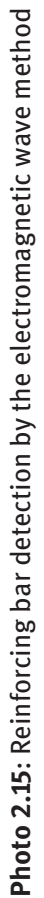
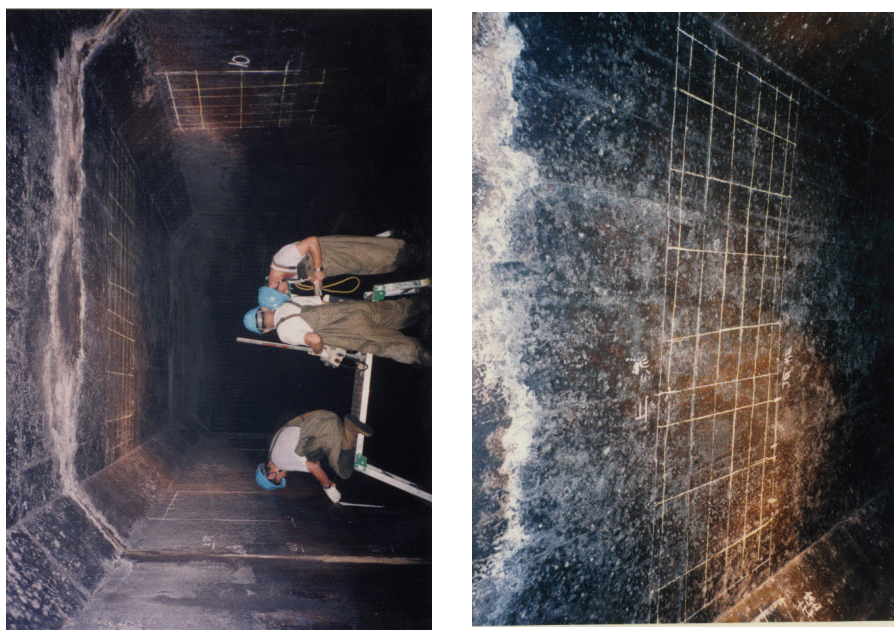
The scanner sends an alternating current of relatively low frequency through the coil in the probe and, by moving along the concrete surface while producing an alternating magnetic field, measures changes in magnetism and displays the results as the distance between the probe (concrete cover) and reinforcing bars. Photo 2.16 shows how reinforcing bar detection is performed by the electromagnetic induction method.

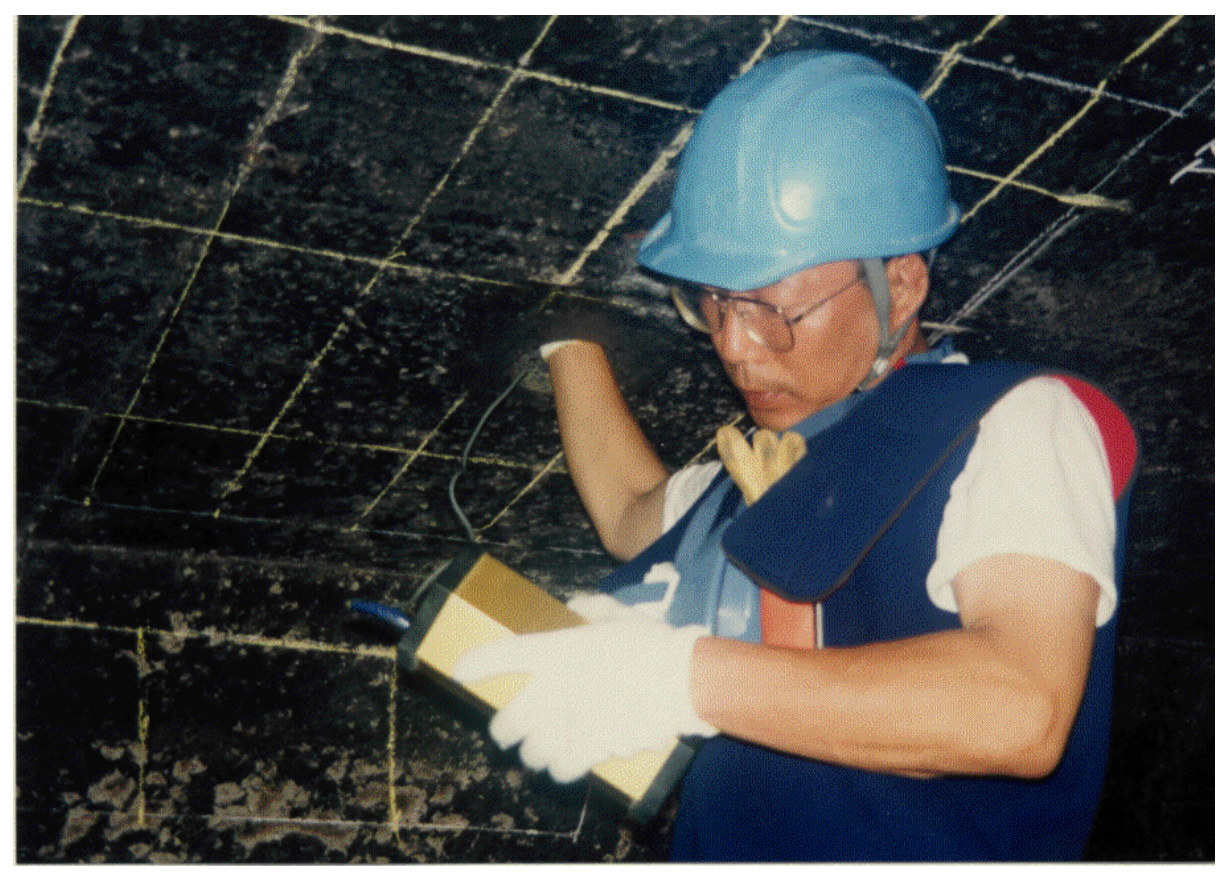

Photo 2.16: Rebar detection by the electromagnetic induction method

4) Rebar corrosion investigation

Concrete is scraped off (about $200 \times 200 \mathrm{~mm}$ ) until the reinforcing bars are exposed, and the concrete cover and the reinforcing bar diameter are measured. Photos 2.17 and 2.18 show how this investigation is carried out.

\section{5) Rebar tension test}

Exposed and corroded portions of reinforcing bars are cut off, and the samples thus obtained are used to measure the corrosion loss ratio and tensile strength. The reinforcing bar samples are immersed in a $10 \mathrm{w} / \mathrm{v} \%$ solution of diammonium hydrogen citrate containing 2-mercaptobenzothiazole (150 ppm) to remove corrosion products. Photos 2.19 and 2.20 show examples of reinforcing bar samples. Following the measurement of the corrosion loss ratio, the yield point and tensile strength are measured in accordance with JIS Z 2241; refer to Photos 2.21 and 2.22. 


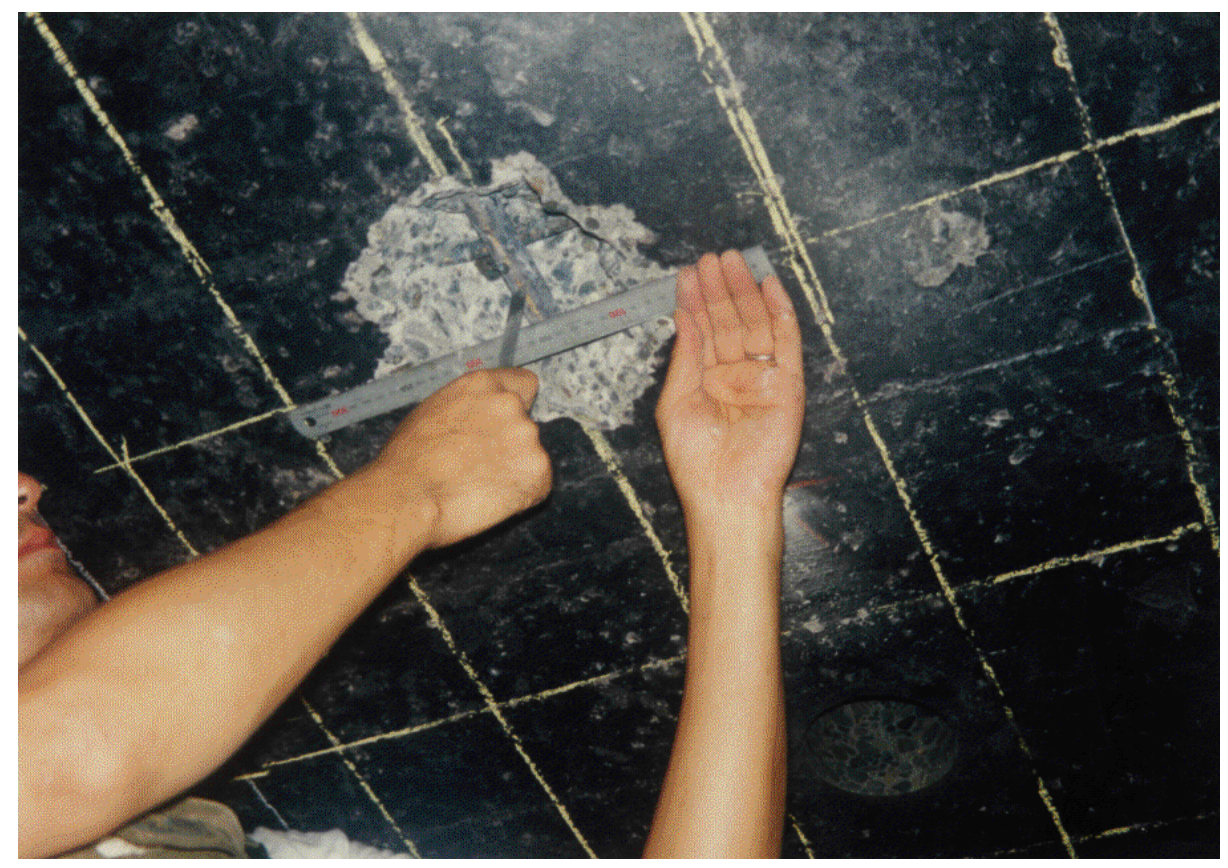

Photo 2.17: Concrete cover measurement

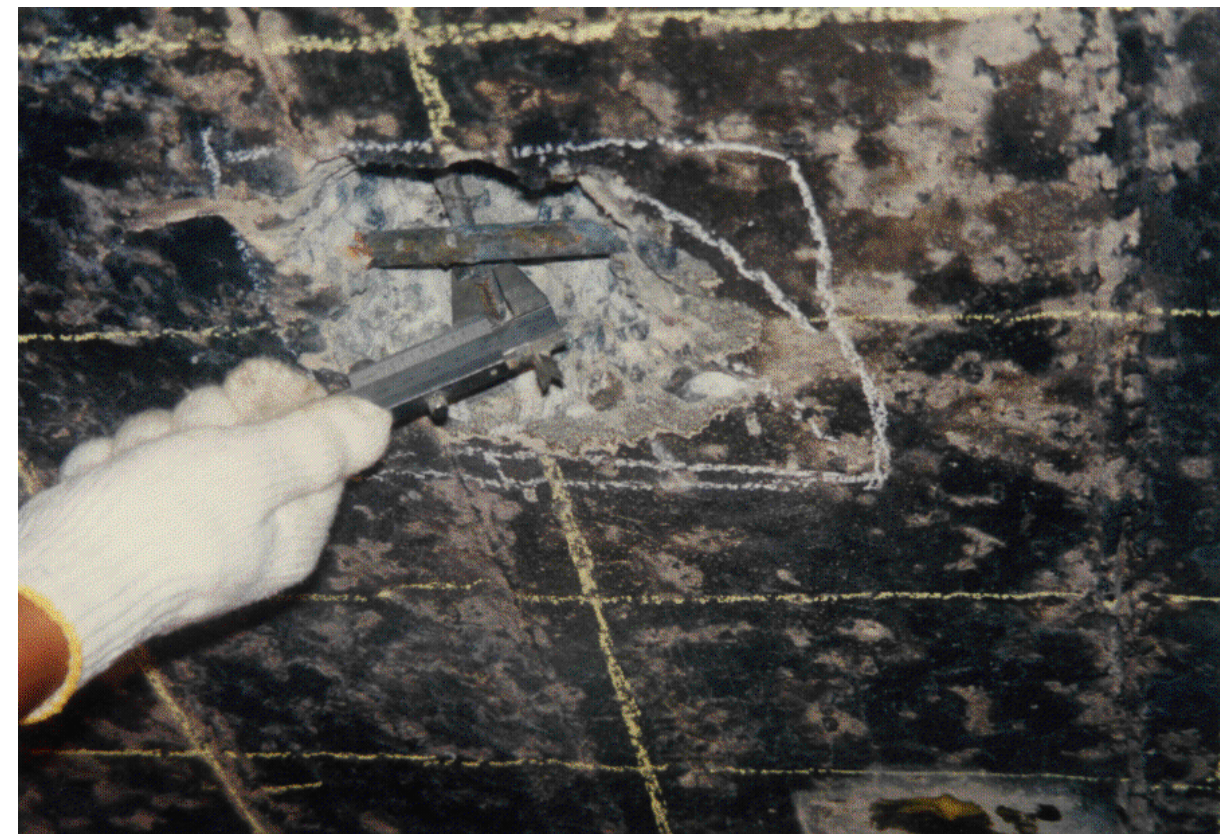

Photo 2.18: Rebar diameter measurement 


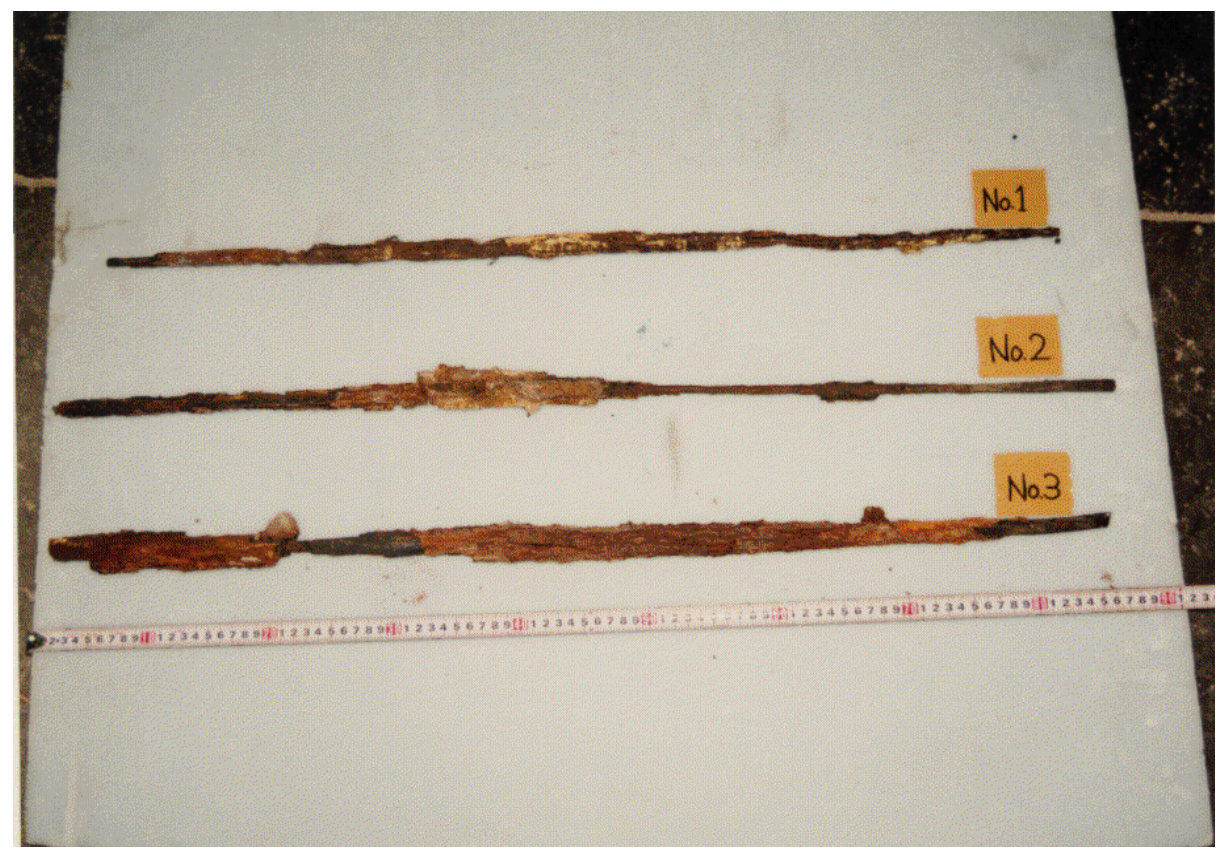

Photo 2.19: Corroded rebar samples

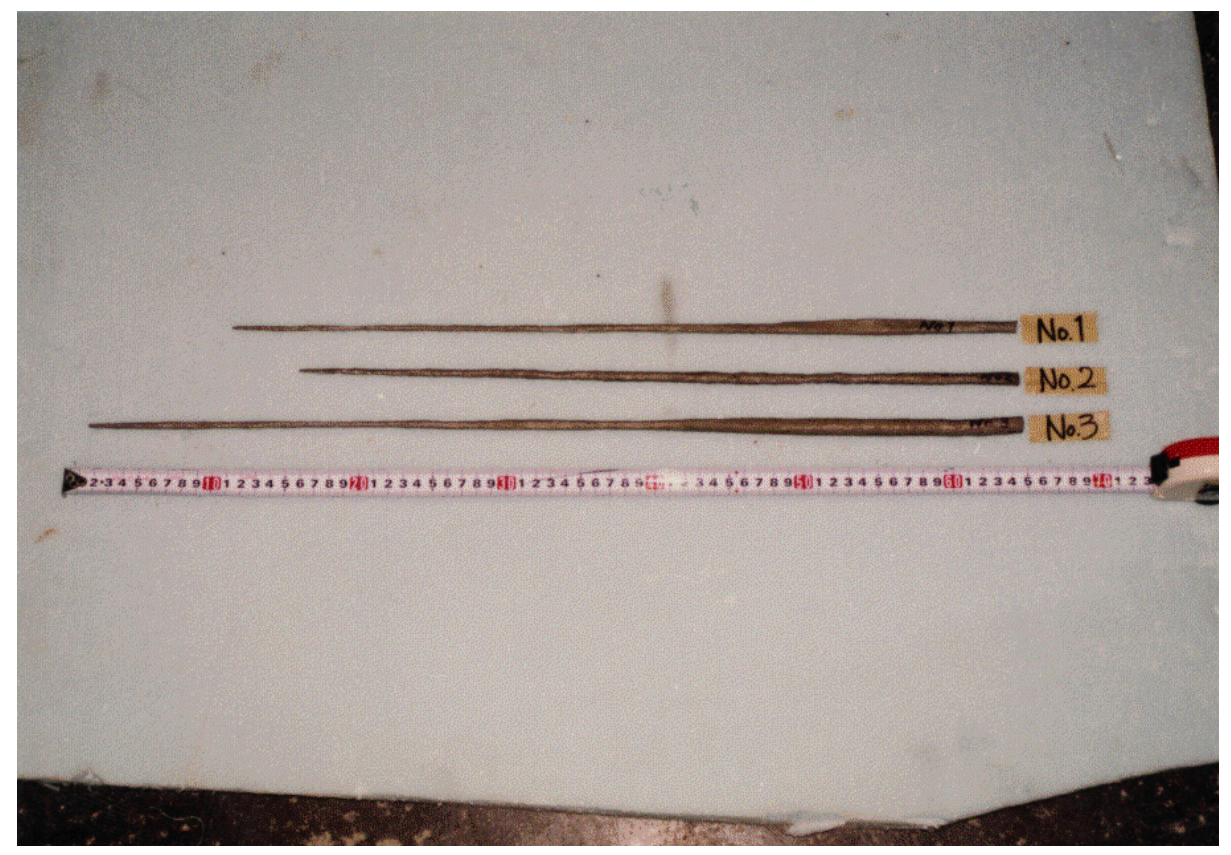

Photo 2.20: Rebar samples after removing corrosion products 


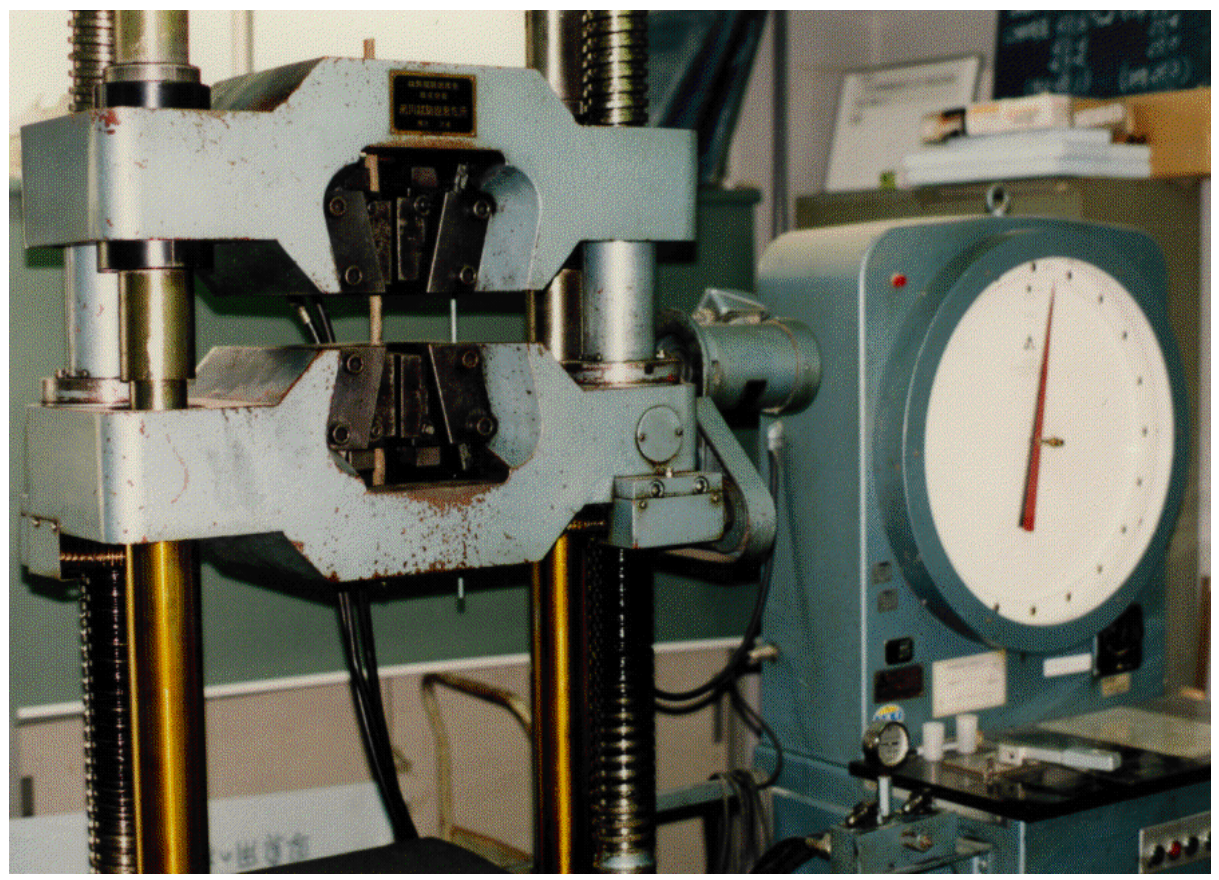

Photo 2.21: Tension test of a corroded rebar

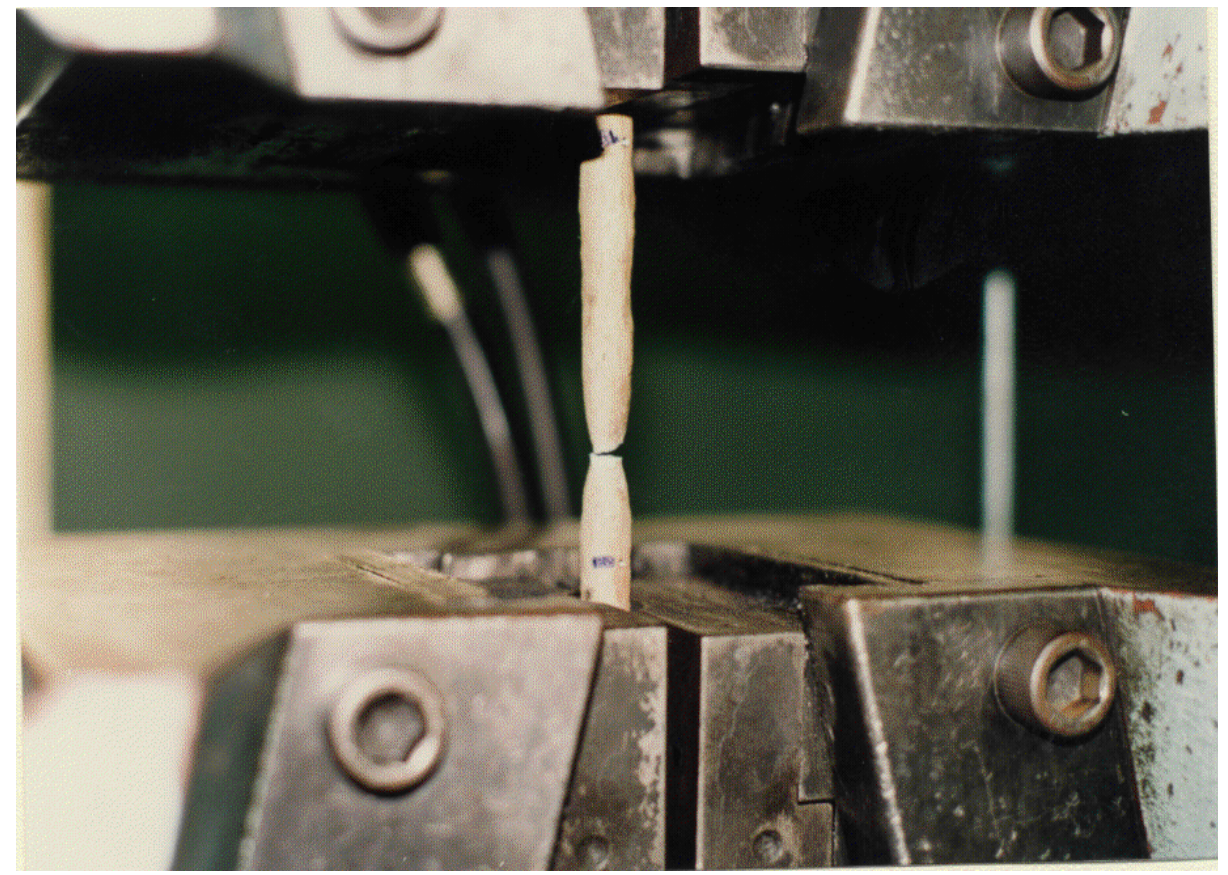

Photo 2.22: Fracture at the tensile strength of a corroded rebar 
6) Structural member thickness investigation

If the design and completion documents of an ageing sewer do not exist, the thickness of concrete members is measured by the ultrasonic method, as shown in Fig. 2.9. The ultrasonic method utilises the fact that ultrasonic waves (sound waves having a frequency of $20 \mathrm{kHz}$ or higher) are elastic waves capable of propagating more easily through a solid than electromagnetic waves, and are reflected at a boundary surface. The thickness of a structural member is calculated as

$$
L=\frac{V}{2 f}
$$

where $L$ : thickness of concrete member ( $\mathrm{mm})$; $V$ : propagation velocity (sound velocity) (about 4,000 m/s in undamaged concrete); $f$ : frequency of reflection $(\mathrm{kHz})$.

As shown in Fig. 2.9, two sensors, one for excitation and another for detection, are attached to the same concrete surface with grease or other similar materials to keep complete contact between the sensors and the surface. Longitudinal ultrasonic waves are transmitted and the received waves are Fourier-analysed with the FFT analyser. The reciprocal of the return time of the reflected wave is shown as the frequency of reflection on the spectrum displayed on the FFT analyser, and the thickness is estimated based on Eq. (2.1). Photos 2.23 and 2.24 show how the thickness measurements are conducted.

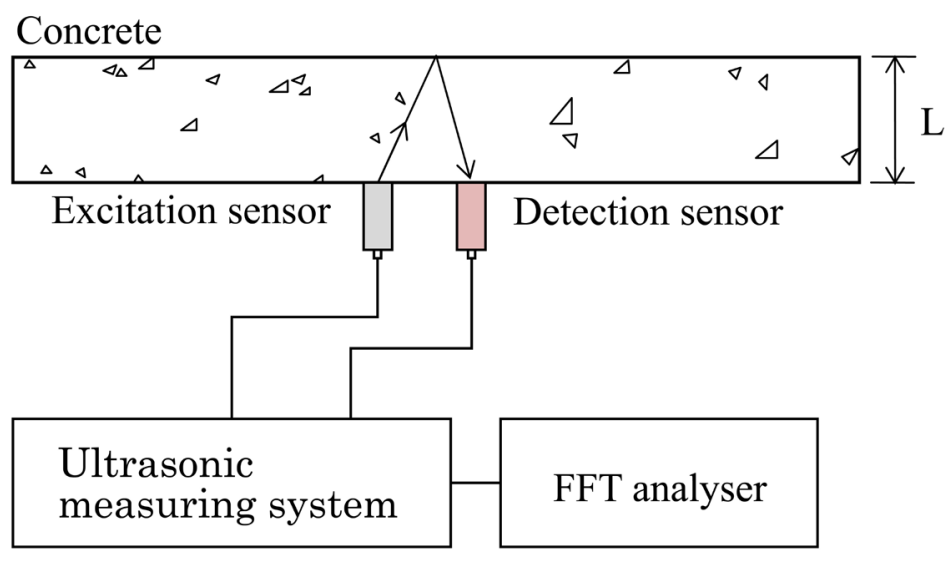

Figure 2.9: Thickness measurement of structural members by the ultrasonic method 


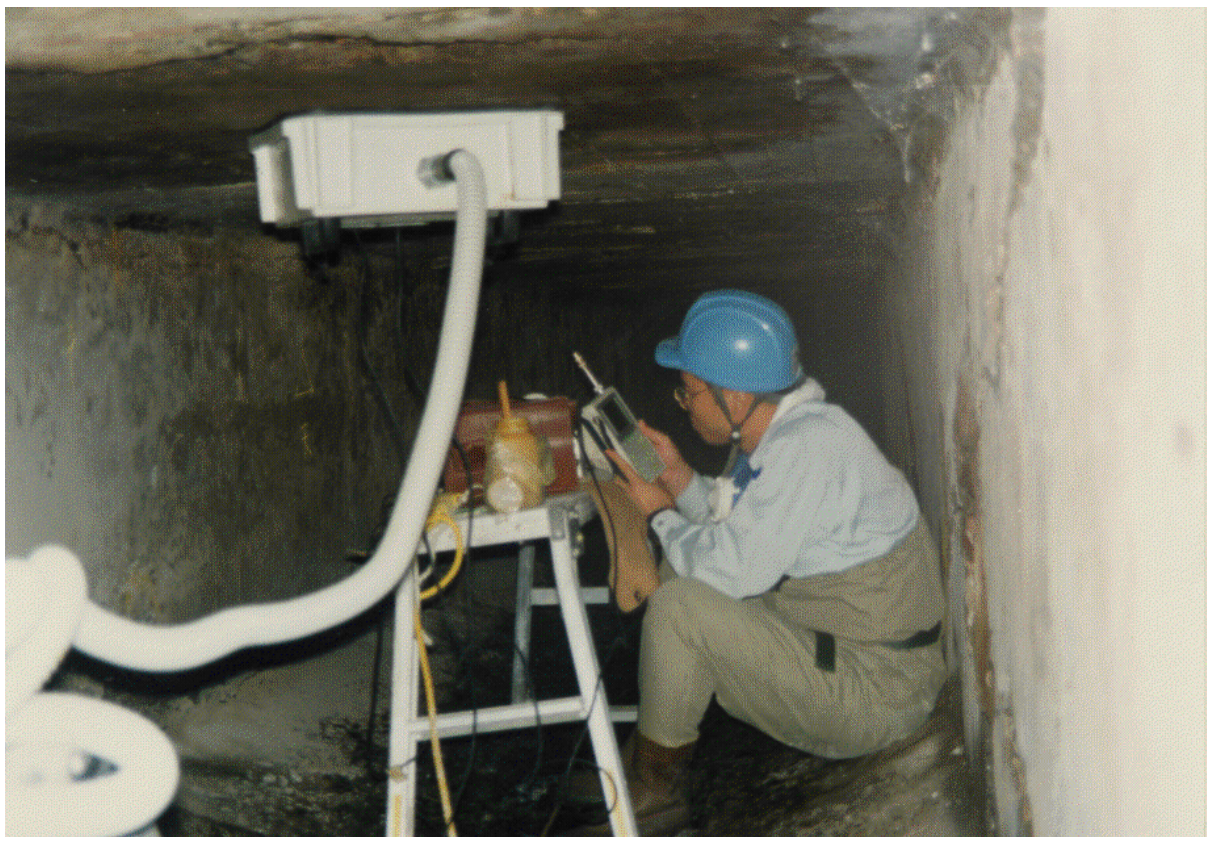

Photo 2.23: Thickness measurement of structural members by the ultrasonic method (1)

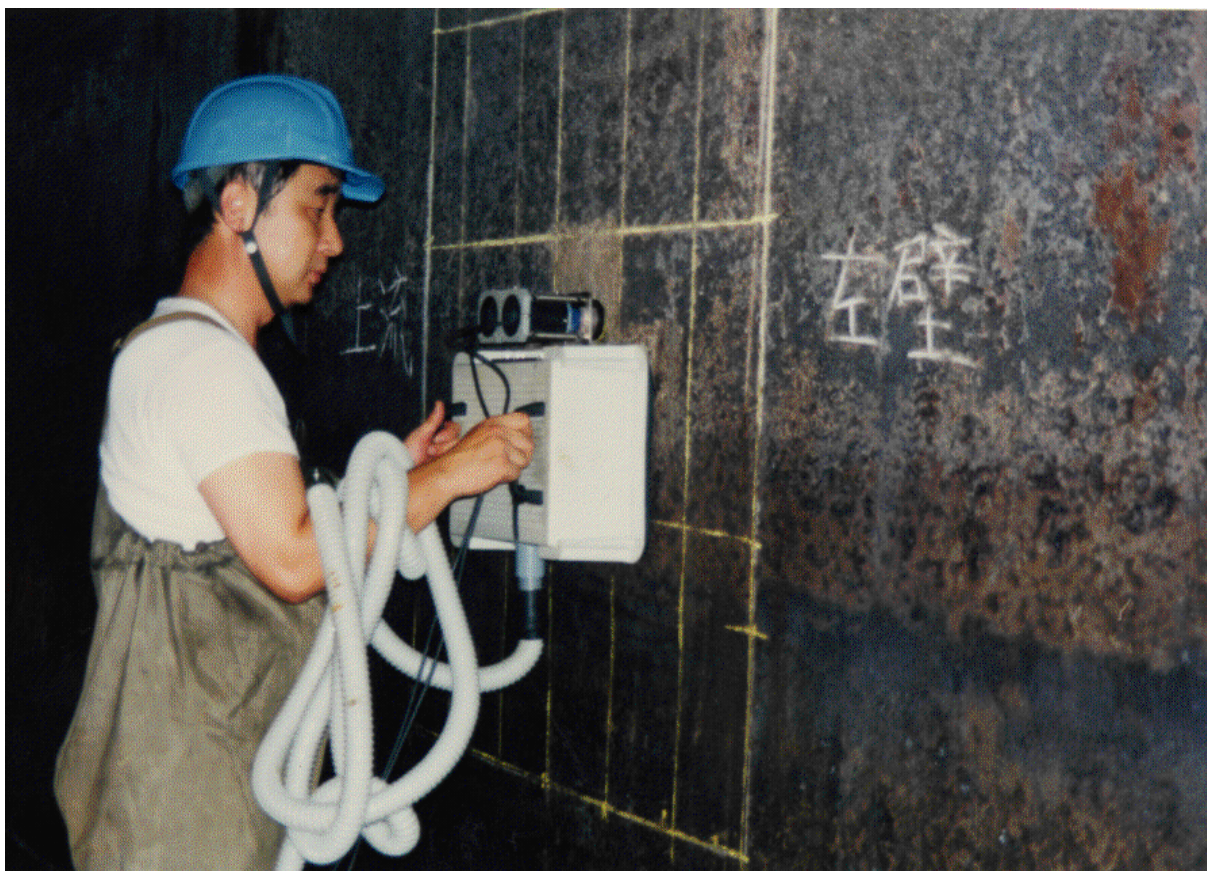

Photo 2.24: Thickness measurement of structural members by the ultrasonic method (2) 


\subsubsection{Small-diameter pipe}

Ageing pipes smaller than $800 \mathrm{~mm}$ in diameter cannot be surveyed from inside by inspectors directly to check on the pipe strength and rebar corrosion. Therefore, for non-man-entry sewers the current design practice for the composite pipe method is to prepare a damaged specimen representing an ageing sewer, renovate it and conduct an external pressure test on the renovated pipe to verify the effectiveness of the renovation method. If the ultimate strength of the renovated pipe exceeds the standardised value of a new pipe, the renovation method is certified as effective and is adopted.

Figure 2.10 presents the comparison results between the fracture strengths of renovated pipes and the standardised strengths and actual test results of new pipes. These fracture tests were performed first on virgin Hume pipes 250 to 3,000 mm in diameter under concentrated line loads. After renovating these damaged pipes by the SPR method, the same fracture tests were carried out again on the renovated pipes (JSWA, 2001). Figure 2.10(a) shows the comparison with the standardised strength of a Hume pipe, and Fig. 2.10(b) shows the comparison with the actual strength of the Hume pipe obtained from the fracture tests.

As shown in Fig. 2.10, the fracture strength of the renovated pipes exceeds both the standardised values and the actual strength values of virgin Hume pipes, and this tendency is particularly noticeable for small-diameter pipes. These results clearly indicate that an ageing pipe with cracks and exposed reinforcing bars can be rehabilitated into a composite pipe stronger than the original one, thus verifying the effectiveness of the renovation method in rehabilitating small-diameter ageing pipes.

The damaged pipe specimens before renovation in Fig. 2.10 had reached the stage of reinforcement yielding, but the rebars had not broken. This damage state should serve as the limit state of damage when applying the renovation method to rehabilitate small-diameter ageing sewers. To evaluate the degree of damage of existing pipes, a travelling mirror-type TV camera as shown in Photo 2.25 is used by the TMG. This TV camera can be used for pipe diameters of 150 to $700 \mathrm{~mm}$ and, when moving forward, is capable of simultaneous shooting in the forward and lateral directions. By adding a pipe interior imaging system and a pipe inspection and diagnosis auxiliary system to the mirror-type TV camera system, the TMG has developed a systematic procedure for producing an expanded drawing of the pipe interior based on the image data and making a quick judgement on the condition of the pipe.

Figure 2.11 shows an example of automatic diagnosis based on the expanded drawing generated from image data. The entries shown in red indicate Level A damage as shown in Table 1.2, and the entries shown in black indicate Level B or Level $\mathrm{C}$ damage as specified in the same table. The entries shown in green indicate information concerning a lateral pipe. As shown in Table 1.2, Level A damage includes longitudinal cracks with a width of $5 \mathrm{~mm}$ or more, disjointing of pipes and a sag in a pipe equal to or greater than the inside diameter of the pipe. In principle, pipes in such conditions must be replaced rather than rehabilitated. Thus, the mirror-type 
TV camera system and automatic diagnosis system can accurately clarify the interior conditions of a small-diameter pipe, enabling a rational decision on the method of pipe reconstruction to be made.

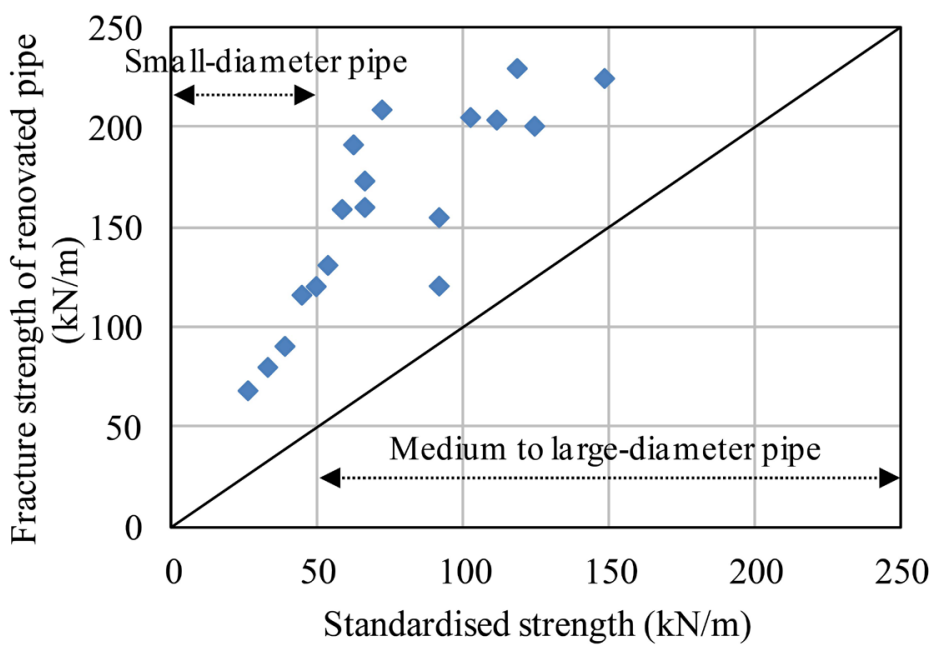

(a)

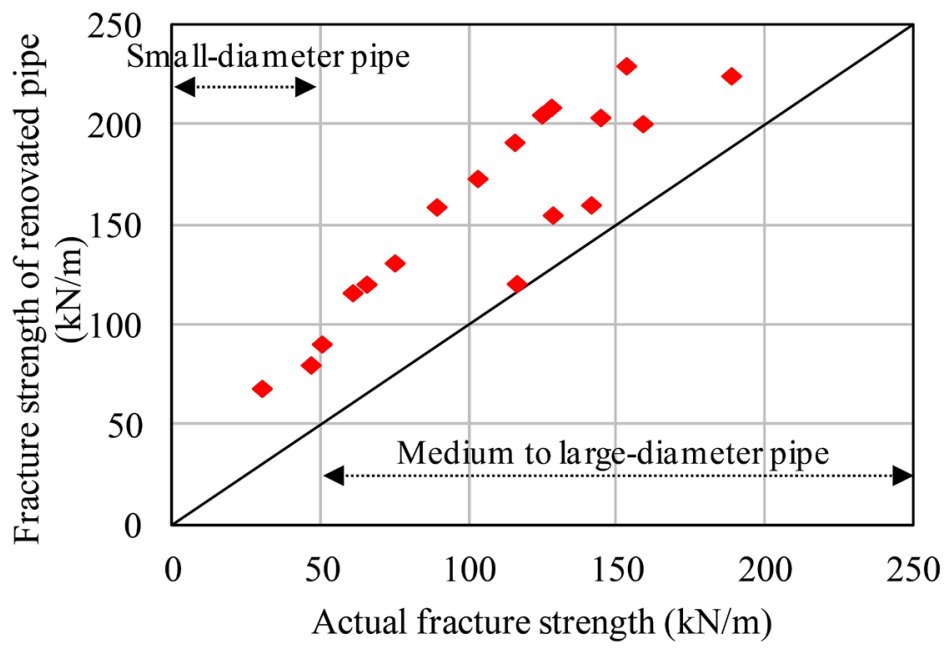

(b)

Figure 2.10: Restoration of fracture strength by pipe renovation: (a) comparison with standardised values; and (b) comparison with actual fracture strength of virgin pipes 

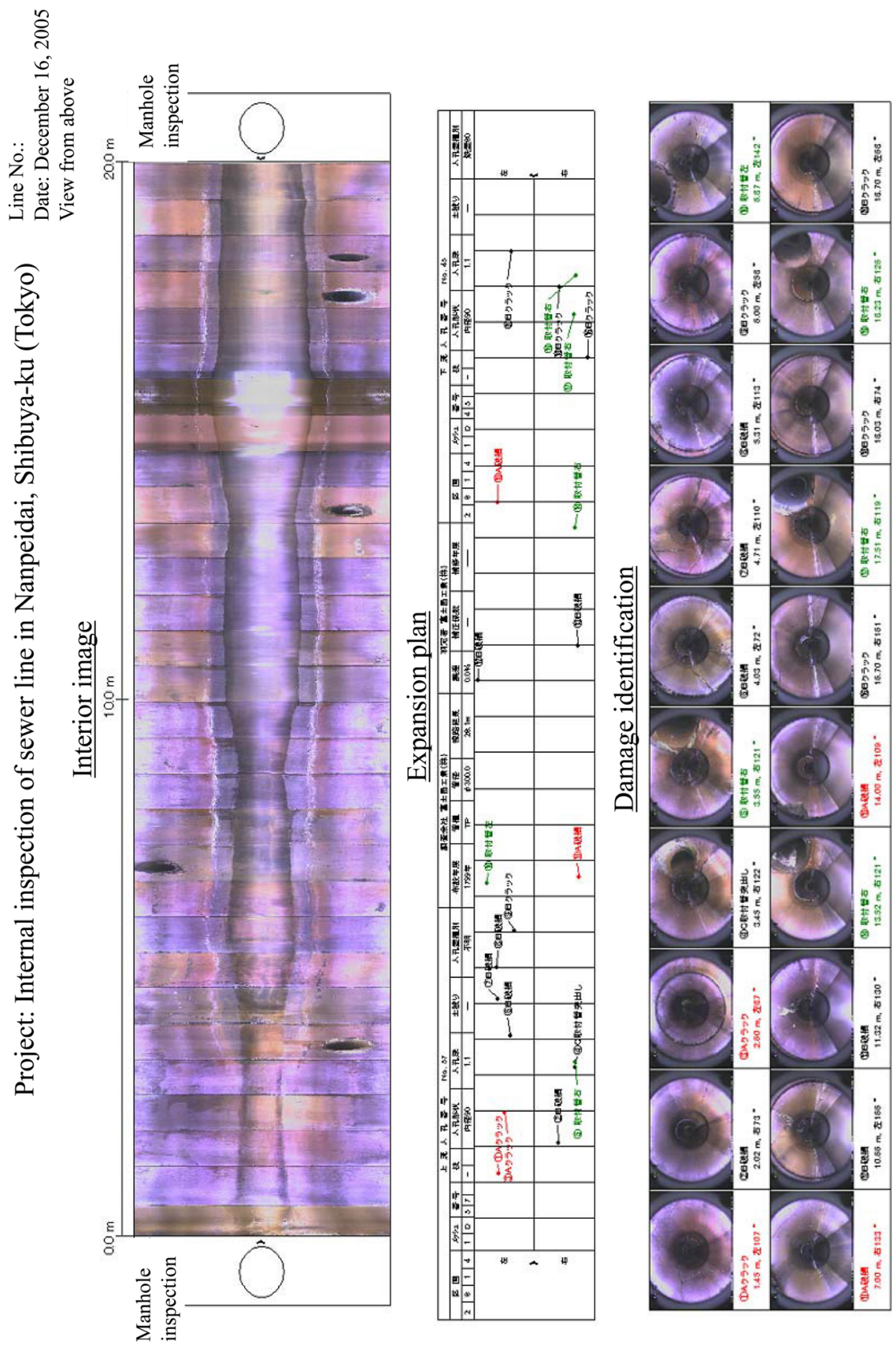

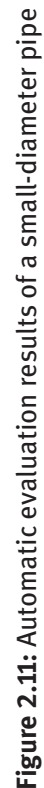




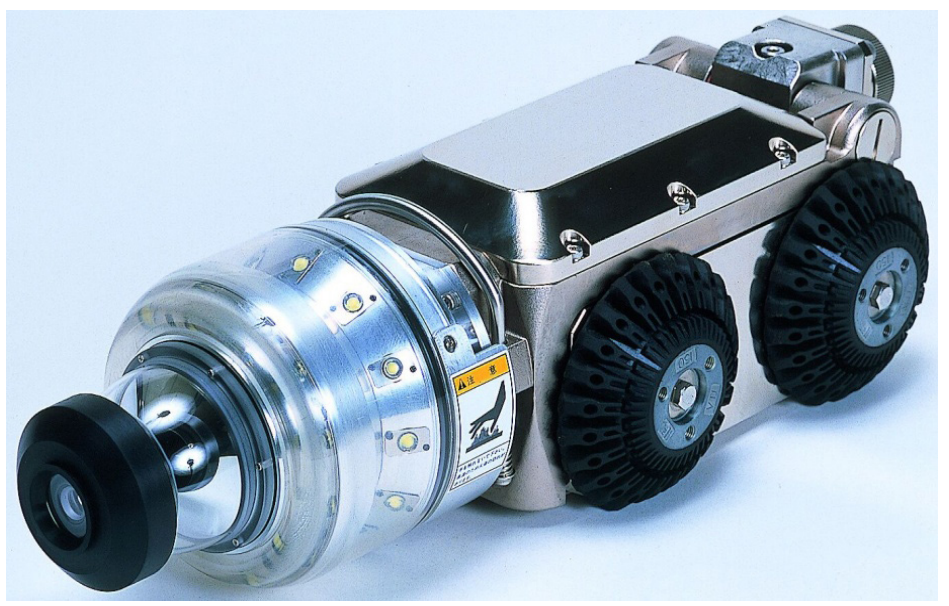

Photo 2.25: A mirror-type TV camera for pipe damage inspection

A number of studies have been carried out to find effective means for evaluating the load-carrying capacity of small-diameter non-man-entry-size pipes. As a promising method in this category, the impact elastic wave method introduced below has been certified in Japan and is now being used in practice (JIWET, 2012b). In this method, an impact is applied to the inside surface of a pipe from one end as shown in Fig. 2.12, and the elastic wave is detected at the opposite end of the pipe. As shown in Fig. 2.13, the frequency spectrum is examined to determine whether high-frequency components have been reduced (reduced high-frequency components indicate the existence of cracks or a reduced wall thickness) and whether the relationship between the wall thickness and the high-frequency component ratio is linear. A system for evaluating the load-carrying capacity of ageing pipes by applying this method has been developed. Photo 2.26 shows an inspection robot consisting of an impactor unit and a receiver unit. At present, the accuracy of the method is still being improved by accumulating inspection data.

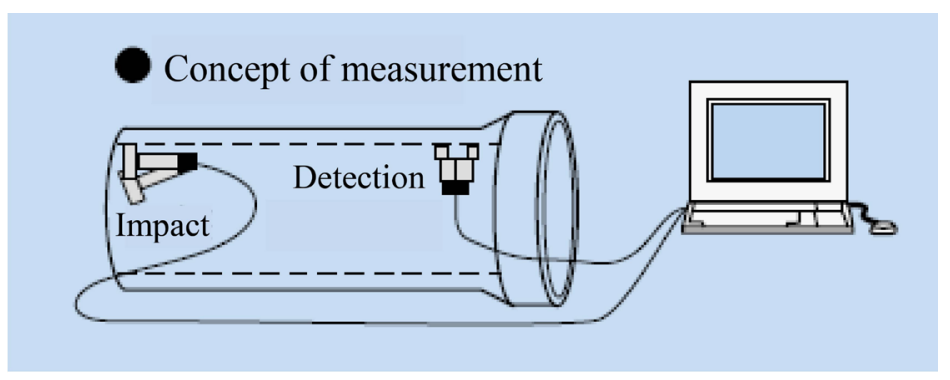

Figure 2.12: The impact wave method 


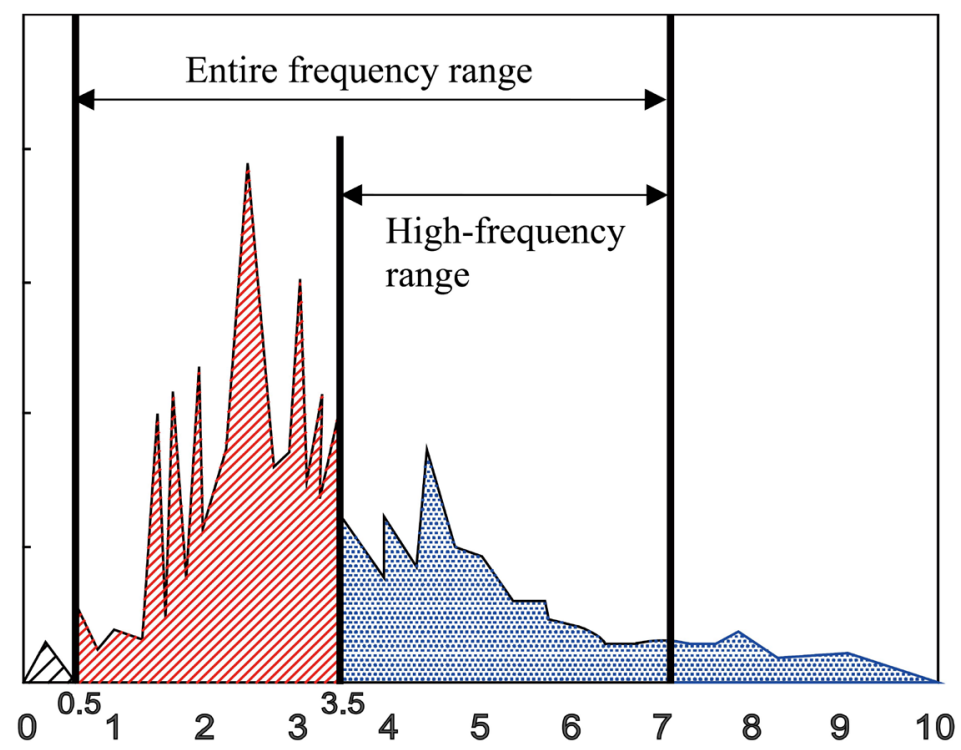

Figure 2.13: Frequency characteristics

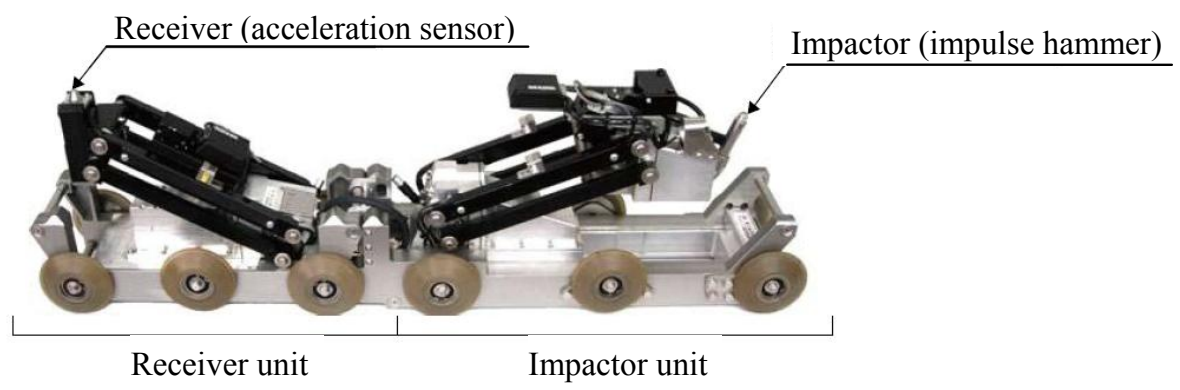

Photo 2.26: An inspection robot of the impact wave method

\subsubsection{Design flow}

Upon completion of the field investigation on an ageing sewer, the cross section of the renovation layer is determined by nonlinear structural analysis based on the limit state design concept, which will be discussed in Chapters 5 and 6. This section presents a brief outline of the design flow, as shown in Fig. 2.14. 


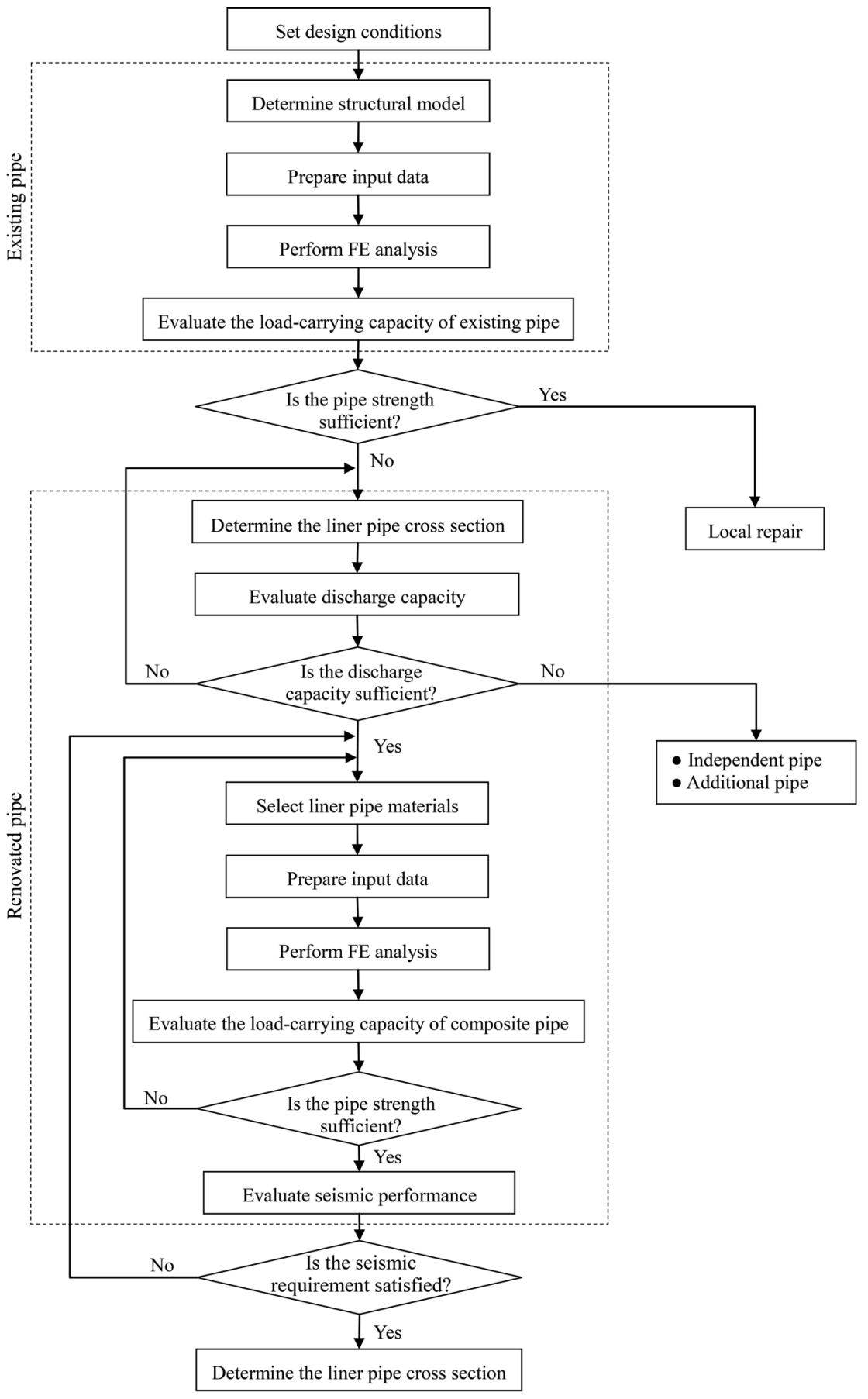

Figure 2.14: Outline of the design flow 
First, the load-carrying capacity of the existing pipe is assessed, and if the strength of the pipe is judged to be insufficient, the possibility of pipe renovation is then evaluated through a computational procedure that takes into account several important factors. If the load-carrying capacity of the existing pipe meets the code requirement, the damaged portions should be repaired locally. Note that there are cases when the insufficient load-carrying capacity of an existing pipe is not caused by pipe deterioration or damage, but by a change in the design standards or safety factors. In such cases the discharge capacity of the pipe is likely to be insufficient, too.

Second, the cross section of the liner pipe is determined so as to meet discharge capacity requirements. If the discharge capacity is insufficient because of an increase in the flow rate, an independent pipe whose liner pipe minimises the cross-sectional reduction will be adopted. If the discharge problem persists, the construction of additional pipes should be considered. After the internal cross section of the renovation layer is determined, specifications for liner pipe materials (in the SPR method, for example, profile strip, reinforcing steel and mortar) are set, and a seismic performance evaluation is carried out to ensure the renovated pipe meets the code requirements for earthquake resistance.

\subsubsection{Construction flow of the SPR method}

Figure 2.15 illustrates the construction flow of the SPR method as an example for the pipe-reforming method; the main procedures are explained below (JSPRA, 2009).

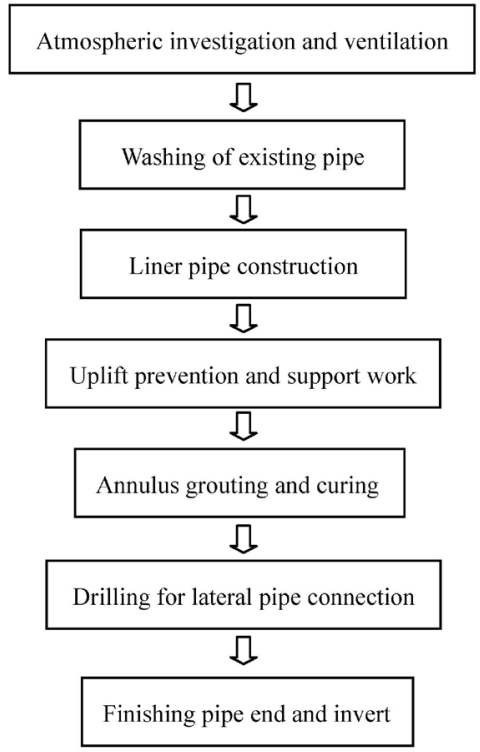

Figure 2.15: Construction flow of the SPR method 
(1) Washing the existing pipe

After measuring the concentration of oxygen and toxic gases in the pipeline and ventilating it, the inside of the pipe is washed to ensure structural integrity between the grout and the existing pipe. In the case of a small-diameter pipe, a spray nozzle attached to the front opening of a high-pressure hose is advanced in the pipe while the nozzle sprays water so as to stir the sludge in the sewer as shown in Fig. 2.16(a). Then, as shown in Fig. 2.16(b), the nozzle is slowly pulled backward, collecting and removing the sludge. In the case of a medium- or large-diameter pipe, as shown in Fig. 2.17, workers enter the pipe and wash its inside by using a spray-gun type washer, brushes, etc.

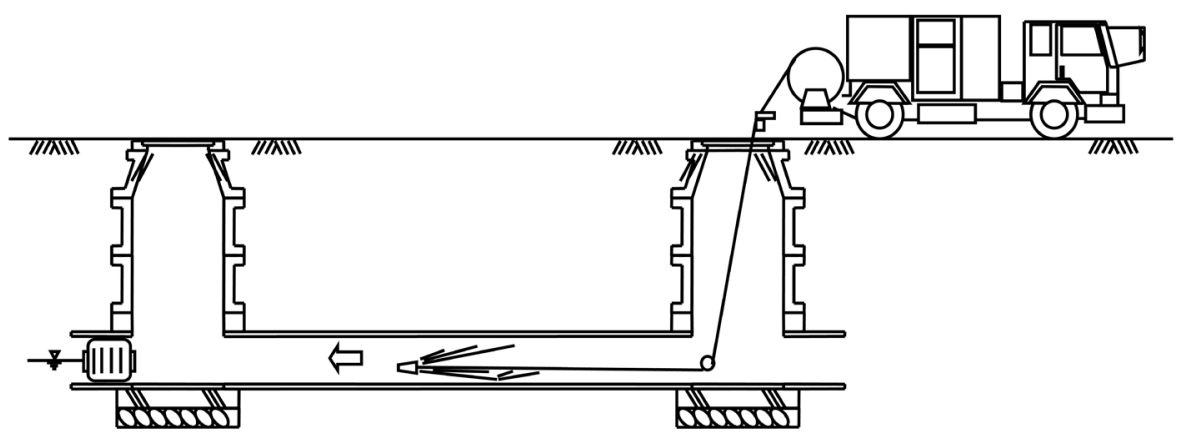

(a)

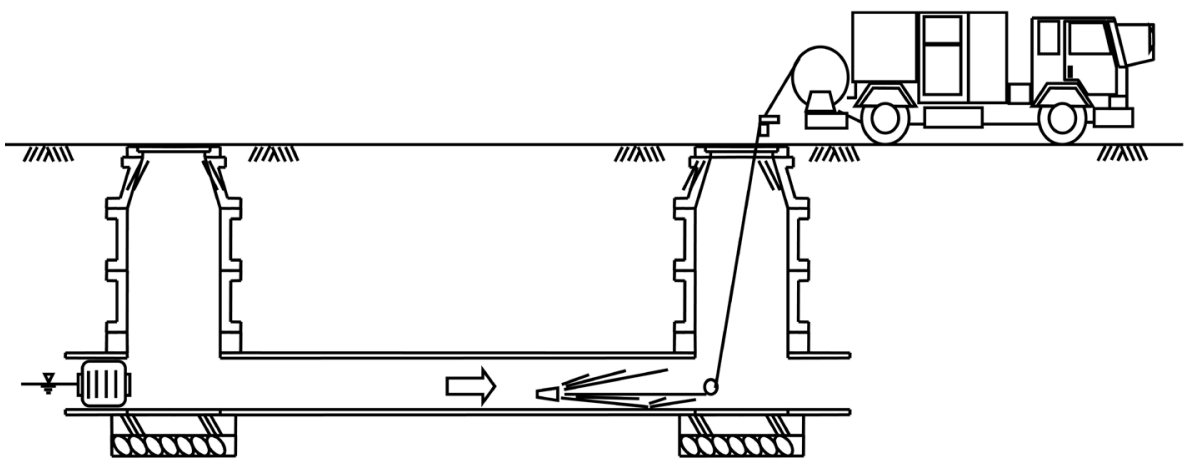

(b)

Figure 2.16: Washing a small-diameter pipe: (a) moving the nozzle forward; and (b) pulling the nozzle back 


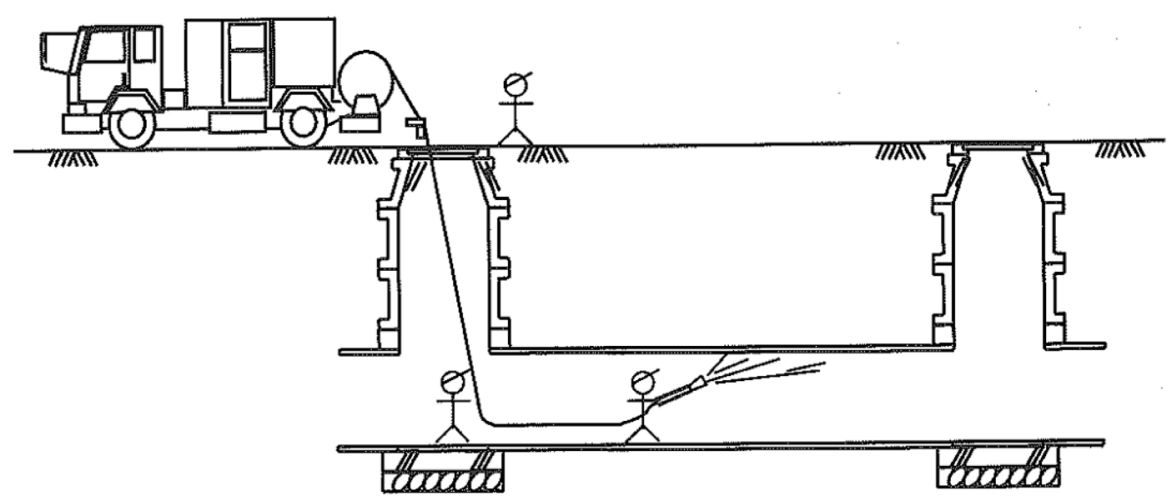

Figure 2.17: Washing a medium- and large-diameter pipe

(2) Liner pipe construction

Figure 2.18 illustrates the jacking method of liner pipe construction used for smalldiameter pipes and the travelling winder method used for medium- and largediameter pipes. In both methods, the winder is disassembled and brought into a 600-mm-diameter manhole and assembled in the manhole. The profile strip is fed from an outer winding drum in the jacking method and from an inner winding drum in the travelling winder method.

In the jacking method, a winding roller is operated in the manhole, and winding is continued while performing strip interlocking and pushing the newly-formed interlining forward until the front tip of the liner pipe arrives at the manhole used as the receiver shaft.

In the travelling winder method, a hydraulic unit is set up in the pipe and a winding roller moves along the pipeline, forming the liner pipe by winding and interlocking the profile strip, which is fed to the winding machine spirally from the profile drum above the ground. Winding is continued until the winder arrives at the manhole used as the receiver shaft.

(3) Uplift prevention and support work

Prior to annulus grouting, uplift prevention and support work is carried out to prevent uplift due to the uplift pressure acting on the liner pipe. Figure 2.19 illustrates how to carry out uplift prevention work for a small-diameter pipe while keeping the sewer in service. Weights such as metal chains are spread in the liner pipe and water is poured into the pipe to prevent uplift.

For a medium- to large-diameter pipe, braces as supports are installed to prevent uplift and the buckling of the liner pipe due to the grouting pressure. Figure 2.20 shows examples of 6-point and 8-point bracings. In order to prevent the uplift of the liner pipe, holes are drilled at the top of the liner pipe so that the braces are supported by the existing pipe. 


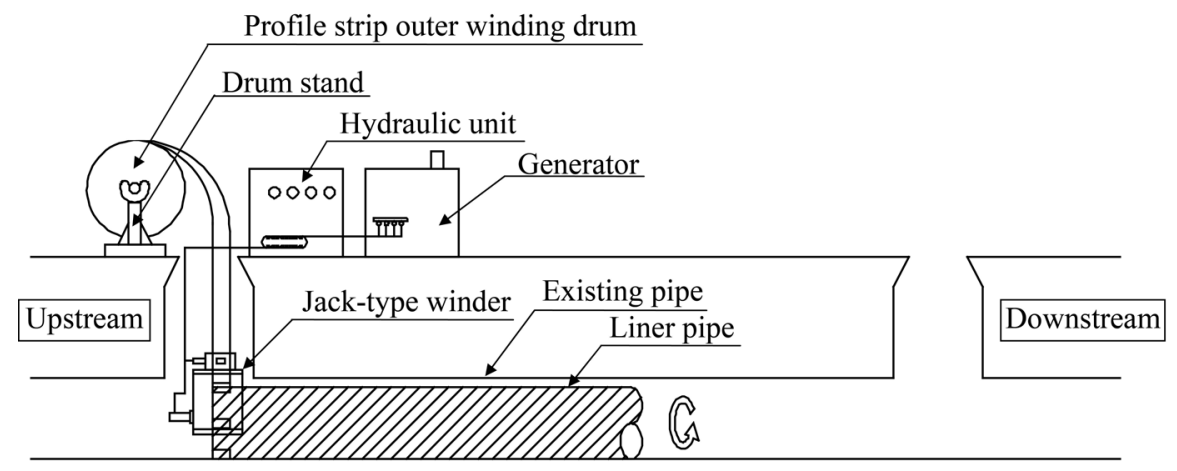

(a)

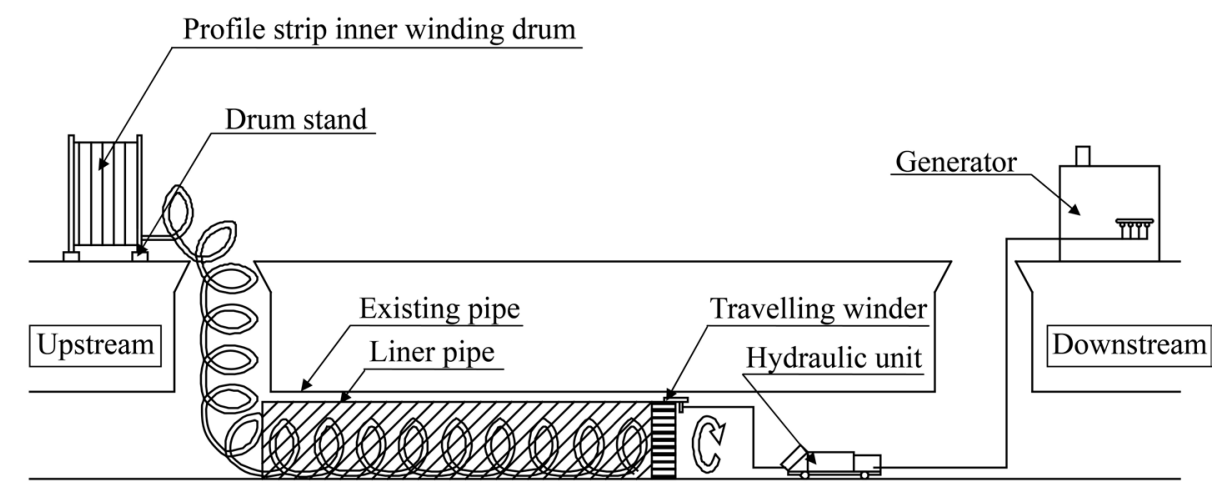

(b)

Figure 2.18: Liner pipe construction systems: (a) jacking method of pipe-making; and (b) travelling winder method of pipe-making

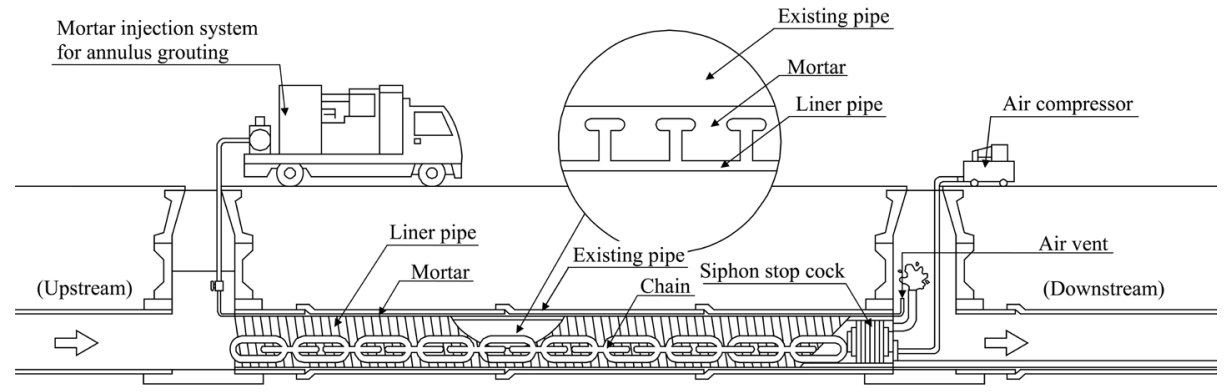

Figure 2.19: Uplift prevention work for small-diameter pipe 


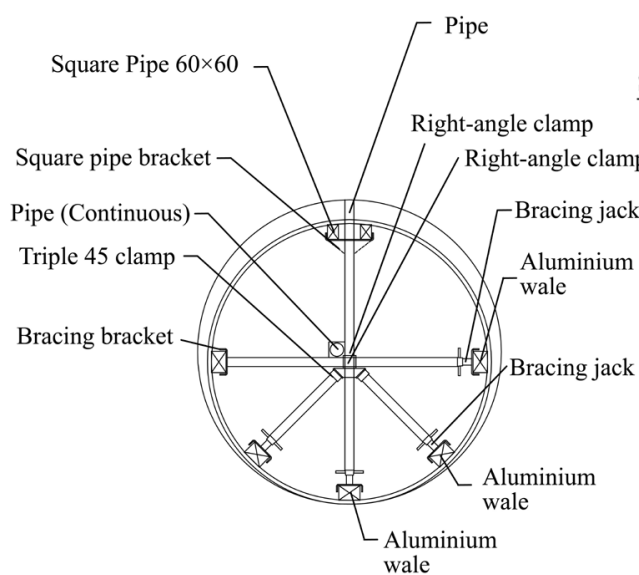

(a) 6-point bracing

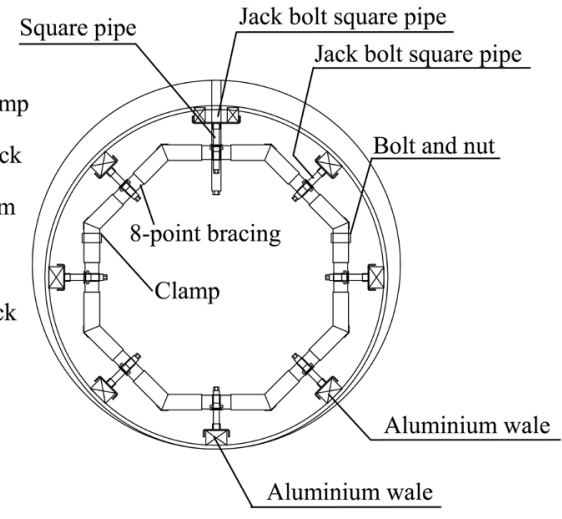

(b) 8-point bracing

Figure 2.20: Uplift prevention framework for medium- and large-diameter pipes

(4) Annulus grouting

In order to prevent grout from flowing into the lateral pipe, an air plug is installed in the lateral pipe inlet from inside the catch basin as shown in Fig. 21. Next, the annular gap between the existing pipe and the liner pipe at the upstream and downstream ends of the pipeline is sealed with a $50 \mathrm{~mm}$-thick layer of clay cement. This sealing is provided with a grout inlet and an air vent. Special mortar is injected from the upstream end, and when the grout spills out of the air vent-cum-overflow valve at the downstream end, the valve is closed. Then, after an overpressure of $0.02 \mathrm{MPa}$ is applied, the inlet valve is closed, and curing is carried out while the overpressure is maintained (Fig. 2.22).

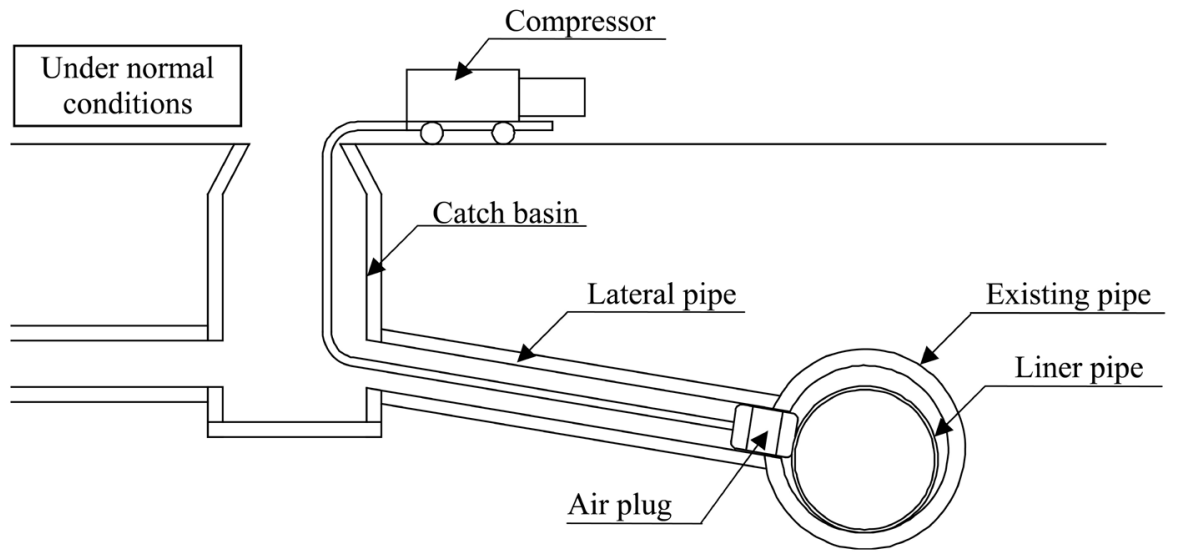

Figure 2.21: Air plugging of lateral pipe 


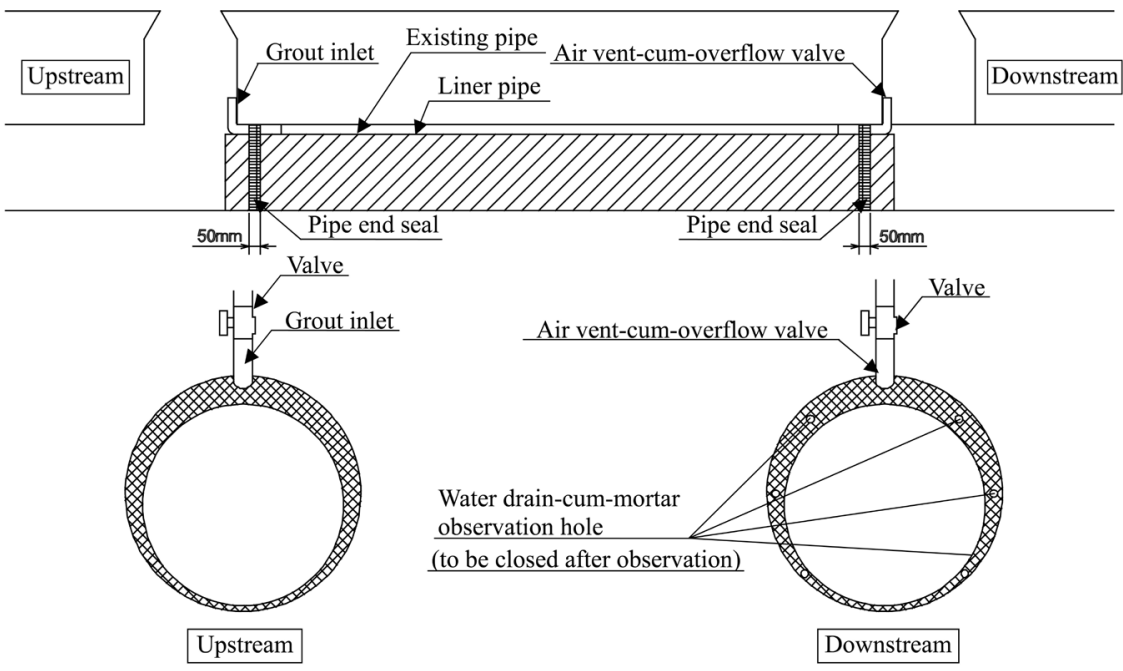

Figure 2.22: Pipe end sealing and grout inlet installation methods

In the case of a small-diameter pipeline, a single span is about $30 \mathrm{~m}$ and the full cross section is grouted in one operation. When dealing with a large-diameter pipeline, however, there may be cases where it is not possible to grout the full cross section in one operation. In such cases as shown in Fig. 2.23, piping (steel pipes) for grouting and grout inlets are provided in the liner pipe, and grouting is carried out in two or more stages.

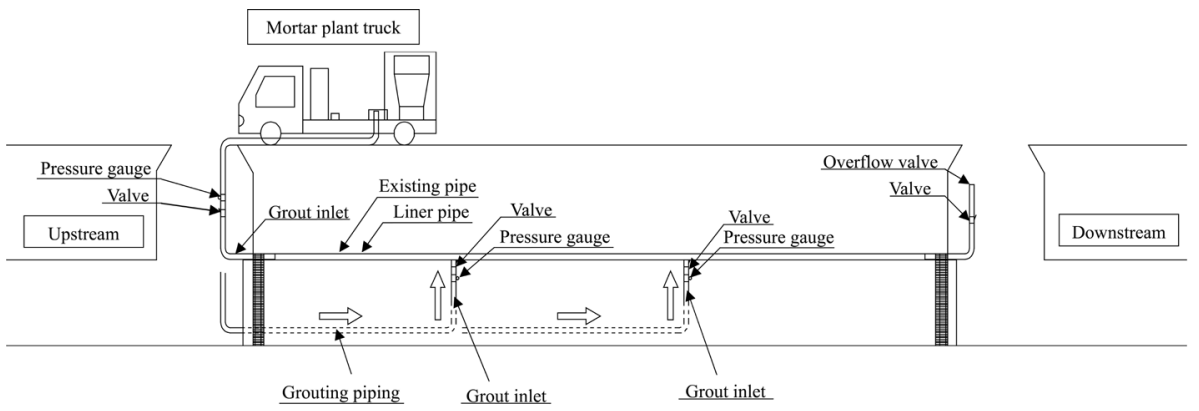

Figure 2.23: Phased grouting in the longitudinal direction

(5) Drilling for lateral pipe connection

After the completion of annulus grouting, drilling for lateral pipe connection is carried out. First, preliminary drilling is performed to determine the drilling location by inserting a drilling machine into the pipe from the catch basin as shown in Fig. 2.24. After locating the pipe position, the main drilling is carried out from inside the 
liner pipe. For small-diameter pipes a robot is used while the drilling operation is monitored through a TV camera as shown in Fig. 2.25; for man-entry pipes it is done manually.

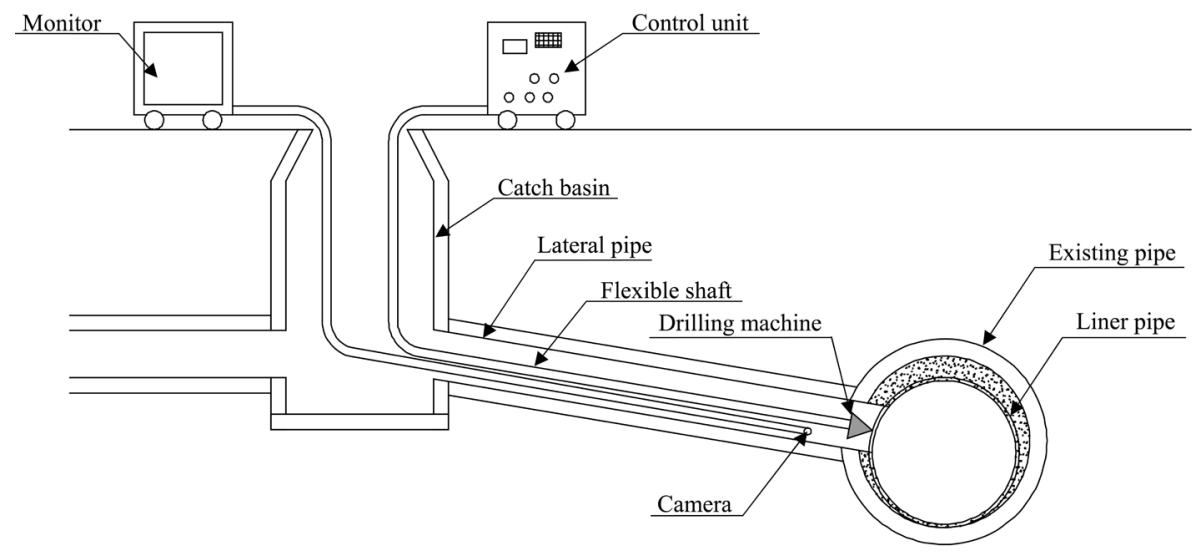

Figure 2.24: Preliminary drilling for lateral pipe connection

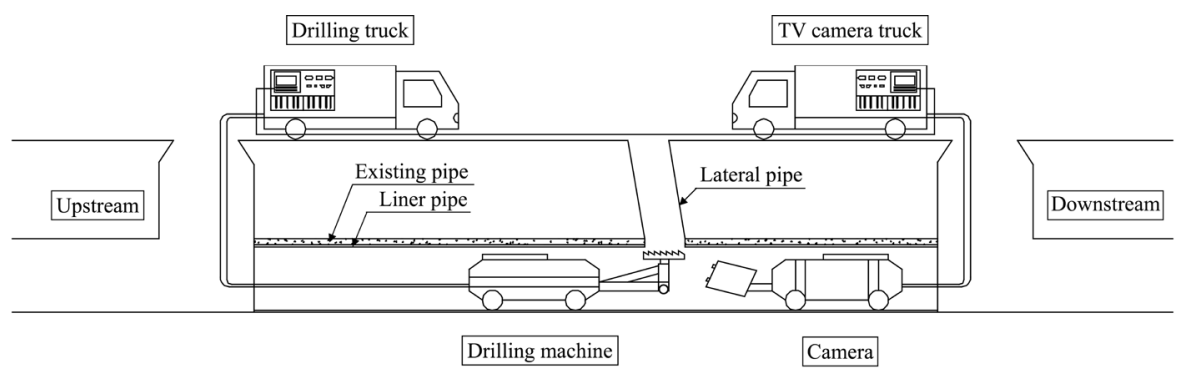

Figure 2.25: Finish drilling for lateral pipe connection

\section{References}

JSWA (2001). Pipe Rehabilitation Handbook. Tokyo, Japan Sewage Works Association.

JIWET (2009a). Construction Technology Certification Report: 3S Segment Method. Tokyo, Japan Institute of Wastewater Engineering Technology.

JIWET (2009b). Construction Technology Certification Report: PALTEM Flow-Ring Method. Tokyo, Japan Institute of Wastewater Engineering Technology.

JIWET (2009c). Construction Technology Certification Report: Danby's Method. Tokyo, Japan Institute of Wastewater Engineering Technology. 
JIWET (2011). Construction Technology Certification Report: PFL Method. Tokyo, Japan Institute of Wastewater Engineering Technology.

JIWET (2012a). Construction Technology Certification Report: SPR Method. Tokyo, Japan Institute of Wastewater Engineering Technology.

JIWET (2012b). Technical Data Book for Pipeline Diagnosis by Impact Elastic Wave Method. Tokyo, Japan Institute of Wastewater Engineering Technology.

JSPRA (2009). SPR Method Construction Manual: Sewer Rehabilitation. Tokyo, Japan SPR Method Association. 